\title{
Adiabatic Sweep Cross-Polarization Magic-Angle-Spinning NMR of Half-Integer Quadrupolar Spins
}

\author{
Sungsool Wi, ${ }^{1 *}$ Chul Kim, ${ }^{2}$ Robert Schurko, ${ }^{3}$ and Lucio Frydman ${ }^{1,4}$
}

${ }^{1}$ National High Magnetic Field Laboratory, Tallahassee, Florida 32304, USA

${ }^{2}$ Department of Chemistry, Hannam University, Taejeon 305811, South Korea

${ }^{3}$ Department of Chemistry and Biochemistry, University of Windsor, 401 Sunset Avenue, Windsor N9B 3P4, Ontario, Canada

${ }^{4}$ Department of Chemical Physics, Weizmann Institute of Sciences, Rehovot, 76100 Israel

* To whom correspondence should be addressed. Email: sungsool@magnet.fsu.edu (S. W.) 


\section{Abstract}

The use of frequency-swept radiofrequency (rf) pulses for enhancing signals in the magic-angle spinning (MAS) spectra of half-integer quadrupolar nuclides was explored. The broadband adiabatic inversion cross-polarization magic-angle spinning (BRAIN-CPMAS) method, involving an adiabatic inversion pulse on the $S$-channel and a simultaneous rectangular spin-lock pulse on the $I$-channel $\left({ }^{1} \mathrm{H}\right)$, was applied to $I(1 / 2) \rightarrow S(3 / 2)$ systems. Optimal BRAIN-CPMAS matching conditions were found to involve low rf pulse strengths for both the $I$ - and S-spin channels. At these low and easily attainable rf field strengths, levelcrossing events among the energy levels $|3 / 2\rangle,|1 / 2\rangle,|-1 / 2\rangle,|-3 / 2\rangle$ that are known to complicate the CPMAS of quadrupolar nuclei, are mostly avoided. Zero- and doublequantum polarization transfer modes, akin to those we have observed for $I(1 / 2) \rightarrow S(1 / 2)$ polarization transfers, were evidenced by these analyses even in the presence of the quadrupolar interaction. ${ }^{1} \mathrm{H}-{ }^{23} \mathrm{Na}$ and $\quad{ }^{1} \mathrm{H}-{ }^{11} \mathrm{~B}$ BRAIN-CPMAS conditions were experimentally explored on model compounds by optimizing the width of the adiabatic sweep, as well as the rf pulse powers of the ${ }^{1} \mathrm{H}$ and ${ }^{23} \mathrm{Na} /{ }^{11} \mathrm{~B}$ channels, for different MAS rates. The experimental data obtained on model compounds containing spin-3/2 nuclides, matched well predictions from numerical simulations and from an average Hamiltonian theory model. Extensions to half-integer spin nuclides with higher spins and potential applications of this BRAIN-CPMAS approach are discussed. 


\section{Introduction}

Cross-polarization magic-angle spinning (CPMAS) ${ }^{1}$ is arguably the most important technique for the characterization of molecular-level structures and dynamics by solid-state NMR. $^{2-7}$ CPMAS relies on the Hartmann-Hahn $(\mathrm{HH})$ condition $^{1,8}$ that arises when the radiofrequency (rf) field applied at the Larmor frequency of a spin-locked nuclide (usually ${ }^{1} \mathrm{H}$ ), matches the rf field applied on either a spin-1/2 or a quadrupolar nuclide, whose sensitivity is to be increased. ${ }^{9-18}$ Although conventional CPMAS involving continuous-wave spin-locking fields on both channels has been effective for carrying out solid-state NMR experiments under moderate MAS rates and magnetic field strengths, this classical approach faces limitations for acquiring NMR spectra that span large bandwidths. This is even more problematic for CPMAS experiments involving quadrupolar nuclei, since the utilization of high rf fields on the quadrupolar channel is often required in order to match the nutation frequency of the spin- $1 / 2$ nucleus. Besides being instrumentally challenging, these high rf fields can induce MAS-driven level crossings among the eigenstates of the quadrupolar nuclide, which in turn will periodically result in the loss of the transferred polarization. ${ }^{19,20}$ Improvements in superconducting magnet technologies ${ }^{21,22}$ have facilitated the solidstate NMR of half-integer quadrupolar nuclei, as the central transition (CT) spectra usually observed in these species are dominated by a second-order quadrupolar interaction that scales the pattern breadth as the inverse of the applied magnetic field. ${ }^{23}$ However, field increases may be associated with requirements to cover wider spectral windows due to larger chemical shift dispersions; this may in turn increase the rf strengths needed to implement the $\mathrm{HH}$ match. The development of CP techniques capable of exciting larger spectral bandwidths has therefore become a topic of active investigation. ${ }^{24-28}$ Frequency-swept rf pulses have often 
been utilized to achieve wider NMR bandwidths, as under power-limited conditions they are known to extend the ranges of excitation, ${ }^{29}$ inversion, ${ }^{30-32}$ decoupling, ${ }^{33,34}$ and refocusing. ${ }^{35,36}$ This has been demonstrated in solution NMR, ${ }^{37}$ in NMR imaging, ${ }^{38}$ as well as in static solidstate NMR experiments on both spin-1/2 and quadrupolar nuclei. ${ }^{24,36,39-43}$

The broadband adiabatic inversion cross-polarization (BRAIN-CP) pulse sequence relies on frequency swept pulses to widen the $\mathrm{HH}$ condition. ${ }^{36,44-47}$ By incorporating Wideband Uniform-Rate Smooth-Truncation (WURST) pulses $^{30}$ for the adiabatic inversion of the rare S-spin polarization, BRAIN-CP can increase the HH matching efficiency over a wide range of isotropic and anisotropic frequencies. While BRAIN-CP was initially utilized under static conditions for obtaining high-sensitivity wideline NMR spectra of spin-1/2 and quadrupolar nuclei, $^{42,43,48-51}$ it has been recently extended to MAS NMR experiments on both spin- $1 / 2^{24}$ and spin-1 nuclides. ${ }^{52}$ In both of these instances, BRAIN-CPMAS has been proved suitable for providing polarization transfer over broad bandwidths - particularly at the very fast MAS spinning rates that will eventually be needed for high field experiments. In this study, we extend the application of the BRAIN-CPMAS method to half-integer quadrupolar nuclei. The behavior of the adiabatic pulses used in the BRAIN-CP sequence is first examined for half-integer quadrupolar nuclides under MAS, in order to understand their spectral inversion properties. In particular, we discuss the effects of the adiabatic sweep on the central, satellite and triple-quantum transitions, and on the conditions determining the Hartmann-Hahn match in the presence of second-order quadrupolar interactions. An average Hamiltonian analysis of the BRAIN-CPMAS method is given, with approximations explaining the zero-quantum (ZQ) and double-quantum (DQ) CP modes affecting the CT spectra. The prospects of using BRAIN-CPMAS are also explored with numerical 
simulations, and with solid-state NMR experiments on model compounds containing ${ }^{23} \mathrm{Na}$ and ${ }^{11} \mathrm{~B}$. These investigations demonstrate that although physically complex and not always providing the optimal route to maximize sensitivity, the BRAIN-CPMAS method can deliver quality powder NMR lineshapes for spin-3/2 nuclides. Furthermore, when strong I-S dipolar couplings are present and the quadrupolar broadening becomes sizable, BRAIN-CPMAS appears to provide a method of choice to collect sensitivity-enhanced CT NMR patterns from these nuclides.

\section{Theoretical Background}

\subsection{Adiabatic inversion behavior of a half-integer quadrupolar spin $S=3 / 2$ under MAS}

The inversion properties of adiabatic frequency-swept pulses have been explored and understood for spin-1/2 solids under both static and spinning conditions. ${ }^{30-32,53,54} \mathrm{~A}$ few examples of extending these considerations to quadrupolar spin- 1 and half-integer spins have also been published. ${ }^{55-58}$ Here, we focus on the inversion behavior arising when a frequencyswept pulse is applied on an ensemble of half-integer quadrupolar spins. We begin by considering the Hamiltonian of an isolated half-integer $(S \geq 3 / 2)$ nucleus undergoing MAS, on which an adiabatic swept pulse is applied. This Hamiltonian in the rotating frame is given by

$$
H_{S}(t)=H_{C S}+H_{Q, S}^{(1)}(t)+H_{Q, S}^{(2)}(t)+H_{r f}(t),
$$

where $H_{C S}$ is a chemical shift term that, ignoring chemical shift anisotropy (CSA, $\left.\delta_{\mathrm{CSA}}\right)$, is provided by

$$
H_{C S}=\Omega_{S} S_{z}=\left(\omega_{0, S}-\omega_{r f, S}\right) S_{z}
$$

$H_{Q, S}^{(1)}(t)$ is the first-order quadrupolar interaction defined by 


$$
H_{Q, S}^{(1)}(t)=\chi_{Q} R_{2,0}^{Q}(t)\left[3 S_{z}^{2}-S(S+1)\right]
$$

where $\chi_{Q}=C_{Q} / 2 S(2 S-1)=e^{2} q Q / 2 S(2 S-1) \hbar$, and $H_{Q, S}^{(2)}(t)$ represents the second-order quadrupolar interaction defined by

$$
\begin{aligned}
H_{Q, S}^{(2)}(t)=\frac{3 \chi_{Q}^{2}}{\omega_{0, S}} & {\left[R_{2,-1}^{Q}(t) R_{2,1}^{Q}(t) S_{z}\left\{4 S(S+1)-8 S_{z}^{2}-1\right\}\right.} \\
& \left.+R_{2,-2}^{Q}(t) R_{2,2}^{Q}(t) S_{z}\left\{2 S(S+1)-2 S_{z}^{2}-1\right\}\right] .
\end{aligned}
$$

The explicit expressions of $R_{2, m}^{Q}(t),(m=0, \pm 1, \pm 2)$ under MAS are obtained by the usual transformation of the electric-field gradient (EFG) tensor from its principal axis system (PAS) to the laboratory frame as given by $\alpha_{Q}, \beta_{Q}$, and $\gamma_{Q}$ Euler angles: ${ }^{59}$

$$
\operatorname{EFG}(S) \stackrel{\left\{\alpha_{Q}, \beta_{Q}, \gamma_{Q}\right\}}{\longrightarrow} \operatorname{ROTOR} \stackrel{\left\{\omega_{r} t, 54.7^{\circ}, 0^{\circ}\right\}}{\longrightarrow} \operatorname{LAB}
$$

The swept pulse (WURST in this case) Hamiltonian, $H_{r f}(t)$, is given by

$$
H_{r f}(t)=-\omega_{1 S} A(t)\left[S_{x} \cos \Psi(t)+S_{y} \sin \Psi(t)\right]
$$

where $\omega_{1 S}=2 \pi v_{1 S}=-\gamma_{s} B_{1 S}$ is the maximum amplitude of the rf field, $A(\mathrm{t})$ is the rf's amplitude envelope (experimentally set to $\left(1-\cos ^{40}\left[\pi t / t_{p}\right]\right)$, although other power forms are also valid), and $S_{x}$ and $S_{y}$ are the transverse rotating-frame spin angular momentum operators. The phase profile of this chirped pulse is given as

$$
\psi(t)=\frac{\Delta \omega}{2 t_{p}} t^{2}-\frac{\Delta \omega}{2} t+\psi_{0}
$$

where $\Delta \omega$ and $t_{p}$ are the frequency sweep range and duration of the adiabatic pulse, respectively. In order to conveniently view the inversion characteristics of this pulse, the rf Hamiltonian in Eq. (6) can be transformed into a frequency-modulated (FM) frame by $U H U^{-1}+i U \frac{d}{d t} U^{-1}$, where $U=\exp \left[i \Psi(t) S_{z}\right] .30-32$ Since the chemical shift Hamiltonian $H_{C S}$ 
and the first- and second-order quadrupolar Hamiltonians, $H_{Q, S}^{(1)}$ and $H_{Q, S}^{(2)}$, commute with the $S_{z}$ operator, the total Hamiltonian in the FM frame is

$$
H_{S}^{F M}(t)=\Omega_{S}^{\prime} S_{Z}+H_{Q, S}^{(1)}(t)+H_{Q, S}^{(2)}(t)-\omega_{1 S} A(t) S_{x}
$$

where $\Omega_{S}^{\prime}=\Omega_{S}-\omega_{p}(t)$, and $\omega_{p}(t)=d \psi(t) / d t=\left(\Delta \omega / t_{p}\right) t-\Delta \omega / 2$ is an instantaneous offset-frequency originating from the evaluation of the Coriolis term, $i U \frac{d}{d t} U^{-1}$.

The behavior of $\left\langle S_{z}\right\rangle$ for a spin-3/2 site under the action of these adiabatic inversion pulses, was examined for a powdered sample under MAS at $v_{r}=40 \mathrm{kHz}$. Figure 1 compares simulated spectra for a site with $C_{\mathrm{Q}}=e^{2} q Q / \hbar=3 \mathrm{MHz}, \eta_{Q}=0, \delta_{c s a}=0$, and $v_{0}=160.4$ $\mathrm{MHz}\left({ }^{11} \mathrm{~B} @ 11.7 \mathrm{~T}\right)$, obtained by varying both the amplitude $v_{1 S}=\omega_{1 S} / 2 \pi\left(v_{1 S}=8\right.$, 20, and $40 \mathrm{kHz}$ ) and the sweep range $\Delta v=\Delta \omega / 2 \pi(\Delta v=5,20$ and $40 \mathrm{kHz})$ of the inversion pulse while using $t_{p}=8 \mathrm{~ms}$. This pulse was assumed followed by an infinitely strong $90^{\circ}$ "read pulse", and by a period of concurrent free evolution and signal digitization. The center of the pulse's sweep range is matched to the center of the central transition peak; i.e., to its isotropic second-order quadrupolar shift. ${ }^{23,60}$ Under the conditions of $\Delta v=5 \mathrm{kHz}=v_{r} / 8$ and $v_{1 S}=8 \mathrm{kHz}$, the simulated spectrum shows a completely inverted, nearly distortion-free MAS-averaged second-order quadrupolar spectrum (marked red in Fig. 1). However, upon sweeping beyond $\Delta v>5 \mathrm{kHz}$ or if performing the spectral inversion with rf fields $v_{1 S}>8$ $\mathrm{kHz}$, the CT lineshape becomes remarkably distorted. This is very different from the inversion characteristics reported for $S=1 / 2$ or for small- $C_{Q} S=1$ (e.g., ${ }^{2} \mathrm{H}$ ) nuclides, for which ideal inversion characteristics were observed for small $v_{1 S}$ over a much larger range of $\Delta v$ values - as long as the sweep range $\Delta v$ satisfies the $\Delta v \leq 2 v_{r}$ condition. $^{24,52}$ In the current case, an inverted, undistorted spectrum is only obtained if the $\Delta v$ is narrow enough to 
encompass the breadth of the anisotropic centerband of the CT transition, but does not overlap with the neighboring spinning sidebands of the satellite transitions (STs). As soon as both CT and ST sidebands are covered by the same rf sweep, however, clear distortions emerge.

\subsection{Spin-lock behavior of the central transition coherence}

Given the aforementioned behaviour of the adiabatic inversion pulse, we now consider the spin-lock behavior of a central transition coherence (CTC), treating it in terms of a fictitious spin-1/2 formalism. ${ }^{61,62}$ Eq. (8) is rewritten as

$$
H_{S}^{F M}(t)=H_{\Omega^{\prime}}(t)+H_{Q, S}^{(1)}(t)+H_{r f}^{F M}(t)
$$

$H_{\Omega^{\prime}}(t)$ is a shift-like Hamiltonian that incorporates the chemical shift, the instantaneous offset frequency $\omega_{p}(t)$, and the second-order quadrupolar interaction. In the fictitious spin$1 / 2$ formalism, this term is

$$
H_{\Omega^{\prime}}(t)=\left\{\Omega_{S}^{\prime}(t)+\Omega_{Q, S}^{2-3}(t)\right\} S_{Z}^{2-3}+\left\{3 \Omega_{S}^{\prime}(t)+\Omega_{Q, S}^{1-4}(t)\right\} S_{Z}^{1-4},
$$

where $S_{z}^{2-3}$ and $S_{z}^{1-4}$ are operators associated with the central (CT) and the triple-quantum (TQ) transitions respectively, and $\Omega_{Q, S}^{2-3}(t)$ and $\Omega_{Q, S}^{1-4}(t)$ represent the second-order quadrupolar interactions of these transitions. The first-order quadrupolar and the rf Hamiltonians can be expressed in this fictitious spin-1/2 formalism as

$$
H_{Q, S}^{(1)}(t)=\omega_{Q}(t)\left\{S_{Z}^{1-2}-S_{Z}^{3-4}\right\}
$$

and

$$
H_{r f}^{F M}(t)=\omega_{1 S} A(t)\left\{2 S_{x}^{2-3}+\sqrt{3}\left(S_{x}^{1-2}+S_{x}^{3-4}\right)\right\},
$$

The explicit expressions for $\omega_{Q}(t)$ and $\Omega_{Q, S}^{m-n}(t)(m-n=2-3$ and 1-4) appearing in Eqs. (10)(12) can be obtained by the coordinate transformations in Eq. (5), and are provided in the 
Appendix. Notice that multiple operators are associated with the rf Hamiltonian in Eq. (12): $S_{x}^{2-3}$ for the CT coherence (CTC), and $S_{x}^{1-2}$ and $S_{x}^{3-4}$ for the two STs that mediate communications between the CTC and the triple-quantum coherence (TQC). To facilitate a description of these communications, we consider these Hamiltonians in a tilted frame rotated by an angle $\theta$, defined by ${ }^{61}$

$$
\theta=\tan ^{-1}\left[\sqrt{3} \omega_{1 S} A(t) / \omega_{Q}(t)\right] .
$$

This tilted frame is characterized by an effective rf field, $\omega_{e}(t)$, described by

$$
\sqrt{\omega_{Q}^{2}(t)+3 \omega_{1 S}^{2} A^{2}(t)}=\omega_{e}(t) .
$$

It is possible to transform the Hamiltonian in Eq. (9) into this tilted FM frame by applying the unitary transformation specified by

$$
e^{-i \theta S_{y}^{1-2}} e^{i \theta S_{y}^{3-4}}
$$

to the various terms introduced in Eqs. (10) - (12). This results in:

$$
\begin{gathered}
H_{T}=\omega_{e}\left(S_{z}^{1-2}-S_{z}^{3-4}\right)+\left\{\Omega^{\prime}(t)+\Omega_{Q, S}^{2-3}(t)\right\} e^{-i \theta S_{y}^{1-2}} e^{i \theta S_{y}^{3-4}} S_{z}^{2-3} e^{-i \theta S_{y}^{3-4}} e^{i \theta S_{y}^{1-2}}+ \\
\left\{3 \Omega^{\prime}(t)+\Omega_{Q, S}^{1-4}(t)\right\} e^{-i \theta S_{y}^{1-2}} e^{i \theta S_{y}^{3-4}} S_{z}^{1-4} e^{-i \theta S_{y}^{3-4}} e^{i \theta S_{y}^{1-2}} 2 \omega_{1 S} A(t) \times \\
e^{-i \theta S_{y}^{1-2}} e^{i \theta S_{y}^{3-4}} S_{x}^{2-3} e^{-i \theta S_{y}^{3-4}} e^{i \theta S_{y}^{1-2}}= \\
\omega_{e}\left(S_{z}^{1-2}-S_{z}^{3-4}\right)+\left\{\Omega^{\prime}(t)+\Omega_{Q, S}^{2-3}(t)\right\}\left[\cos ^{2} \frac{\theta}{2} S_{z}^{2-3}+2 \sin \theta S_{y}^{1-2}-\sin \frac{\theta}{2} S_{y}^{3-4}\right]+ \\
\left\{3 \Omega^{\prime}(t)+\Omega_{Q, S}^{1-4}(t)\right\}\left[\cos ^{2} \frac{\theta}{2} S_{z}^{1-4}+2 \sin \theta S_{y}^{1-2}-\sin \frac{\theta}{2} S_{y}^{3-4}\right]+ \\
2 \omega_{1 S} A(t)\left[\cos ^{2} \frac{\theta}{2} S_{x}^{2-3}-\sin ^{2} \frac{\theta}{2} S_{x}^{1-4}+2 \sin \theta S_{x}^{1-3}-2 \sin \theta S_{x}^{2-4}\right] .
\end{gathered}
$$

In obtaining the above equation, we have utilized

$$
\begin{gathered}
{\left[S_{y}^{1-2}, S_{z}^{2-3}\right]=\frac{i}{2} S_{x}^{1-2} ;\left[S_{y}^{3-4}, S_{z}^{2-3}\right]=\frac{i}{2} S_{x}^{3-4} ;\left[S_{y}^{1-2}, S_{z}^{1-4}\right]=\frac{i}{2} S_{x}^{1-2} ;\left[S_{y}^{3-4}, S_{z}^{1-4}\right]=\frac{i}{2} S_{x}^{3-4}} \\
{\left[S_{y}^{3-4}, S_{x}^{2-3}\right]=\frac{i}{2} S_{x}^{2-4} ;\left[S_{y}^{1-2}, S_{x}^{2-3}\right]=\frac{i}{2} S_{x}^{1-3} ;\left[S_{y}^{1-2}, S_{z}^{2-4}\right]=\frac{i}{2} S_{x}^{1-4}}
\end{gathered}
$$


and

$$
\begin{gathered}
{[P, Q]=i \kappa R} \\
e^{-i \theta P} Q e^{i \theta P}=Q \cos (i \kappa \theta)+R \sin (i \kappa \theta) .
\end{gathered}
$$

The rf term in Eq. (16) contains the $S_{x}^{2-3}$ and $S_{x}^{1-4}$ operators that excite the CTC and TQC, respectively, as well as $S_{x}^{1-3}$ and $S_{x}^{2-4}$ operators that excite double-quantum coherences (DQCs). The magnitude of $\theta$, and thus the magnitude of the applied $\omega_{1 S}$ under a given $\omega_{Q}(t)$, must be significant in order to excite these DQC and TQC to an appreciable degree. Notice that all of the $S_{x}^{2-3}, S_{x}^{1-4}$, and $S_{x}^{1-3}$ and $S_{x}^{2-4}$ terms in Eq. (16) are modulated by MAS-driven quadrupolar oscillations; therefore, numerical evaluations are generally required for their explicit analysis.

The evolution imparted by the dominant first-order quadrupolar term in Eq. (16) (the leading term in this expression) can be removed by going into a quadrupolar interaction frame defined by

$$
\widetilde{H_{T}}=U_{Q} H U_{Q}^{-1}+i U_{Q} \frac{d}{d t} U_{Q}^{-1}
$$

where $U_{Q}=T \exp \left\{i \int_{0}^{t} d t^{\prime} \omega_{e}\left(t^{\prime}\right)\left[S_{Z}^{1-2}-S_{Z}^{3-4}\right]\right\}$ and $U_{Q} \frac{d}{d t} U_{Q}^{-1}=0$. Since the $S_{Z}^{2-3}$ and $S_{Z}^{1-4}$ operators in the shift-like Hamiltonians and the $S_{x}^{2-3}, S_{x}^{1-4}, S_{x}^{1-3}$ and $S_{x}^{2-4}$ operators in the rf Hamiltonians commute with $S_{Z}^{1-2}$ and $S_{Z}^{3-4}$ in $H_{Q}^{(1)}(t)$, Eq. (19) becomes

$$
\begin{gathered}
\widetilde{H_{T}}=\left\{\Omega^{\prime}(t)+\Omega_{Q, S}^{2-3}(t)\right\}\left[\cos ^{2} \frac{\theta}{2} S_{z}^{2-3}+\left\{2 \sin \theta S_{y}^{1-2}-\sin \frac{\theta}{2} S_{y}^{3-4}\right\} \cos \int_{0}^{t} \omega_{e}\left(t^{\prime}\right) d t^{\prime}\right. \\
\left.-\left\{2 \sin \theta S_{x}^{1-2}-\sin \frac{\theta}{2} S_{x}^{3-4}\right\} \sin \int_{0}^{t} \omega_{e}\left(t^{\prime}\right) d t^{\prime}\right]+ \\
\left\{3 \Omega^{\prime}(t)+\Omega_{Q, S}^{1-4}(t)\right\}\left[\cos ^{2} \frac{\theta}{2} S_{z}^{1-4}+\left\{2 \sin \theta S_{y}^{1-2}-\sin \frac{\theta}{2} S_{y}^{3-4}\right\} \cos \int_{0}^{t} \omega_{e}\left(t^{\prime}\right) d t^{\prime}\right. \\
\left.-\left\{2 \sin \theta S_{x}^{1-2}-\sin \frac{\theta}{2} S_{x}^{3-4}\right\} \sin \int_{0}^{t} \omega_{e}\left(t^{\prime}\right) d t^{\prime}\right]+
\end{gathered}
$$




$$
2 \omega_{1 S} A(t)\left[\cos ^{2} \frac{\theta}{2} S_{x}^{2-3}-\sin ^{2} \frac{\theta}{2} S_{x}^{1-4}+2 \sin \theta S_{x}^{1-3}-2 \sin \theta S_{x}^{2-4}\right] .
$$

By considering only the CTC and under the condition $\omega_{Q} \gg \omega_{1 S}$, we can approximate $\theta \approx 0$ and $\omega_{e} \approx \omega_{Q}$. Eq. (20) then reduces to

$$
\widetilde{H_{T}}=\left\{\Omega^{\prime}(t)+\Omega_{Q, S}^{2-3}(t)\right\} S_{z}^{2-3}+2 \omega_{1 S} A(t) S_{x}^{2-3} .
$$

Eq. (21) reveals that the rf term for the excitation of the CTC is free from a quadrupolarinduced evolution if $\omega_{Q} \gg \omega_{1 S}$.

Figure 2 further explores these features by showing the behavior of various states associated with a spin-3/2 manifold, under the action of an adiabatic RF pulse. Described here are the fates of the $S_{z}=|+3 / 2\rangle,|+1 / 2\rangle,|-1 / 2\rangle$ and $|-3 / 2\rangle$ populations, as well as the spin-locking behavior of the CTCs and TQCs, as the rf maximum amplitude $v_{1 s}=\omega_{1 S} / 2 \pi$ and the magnitude of the quadrupolar coupling constant $C_{\mathrm{Q}}$, are varied. In all cases, the effect of MAS on the quadrupolar Hamiltonian is accounted for by Eq. (5), and the CSA is ignored for simplicity. For all of the $v_{1 s}$ values considered, $|+3 / 2\rangle,|+1 / 2\rangle,|-1 / 2\rangle$ and $|-3 / 2\rangle$ populations are inverted at the completion of the sweep when $C_{\mathrm{Q}}=0$. However, when $C_{\mathrm{Q}} \neq$ 0, level crossings between the Zeeman eigenstates that are being modulated by MAS-driven quadrupolar oscillations occur during the sweep. This leads to a rapid dissipation of the spinlocked states, particularly when larger $v_{1 s}$ values are used. Smooth CT spectral inversions free from level crossings are only achieved for weak $(<10 \mathrm{kHz}) \mathrm{rf}$ pulse strengths. At these small rf amplitudes, population inversions occur between the central $|+1 / 2\rangle$ and $|-1 / 2\rangle$ energy eigenstates in accordance with Eq. (21), while changes in populations of the outer $|3 / 2\rangle$ and $|-3 / 2\rangle$ energy eigenstates populations are minimal. This suggests that these are the optimal $\mathrm{rf}$ amplitudes to be used for efficient $I\left({ }^{1} \mathrm{H}\right) \rightarrow S(\mathrm{CT} ; S=3 / 2)$ BRAIN-CPMAS (vide infra). When $v_{1 s}>12 \mathrm{kHz}$, fast oscillations and dissipations of polarization between 
the energy states arise, owing to quadrupolar- and MAS-driven level crossings. ${ }^{19,63}$ Introduction of CSA, which we incorporated with values of $\delta_{\mathrm{CSA}}$ up to a few tens of ppm (simulations not shown), had no major influence on this behavior.

\subsection{The BRAIN CPMAS Hamiltonian: Transfer dynamics of the central transition}

The Hamiltonian required to describe the $I(1 / 2) \rightarrow S(3 / 2)$ BRAIN-CP phenomenon under MAS requires adding an $I$-spin rf term as well as an $I$-S dipolar coupling term to the previous expressions. Using Eq. (20) as starting point, the resulting Hamiltonian required for considering the BRAIN-CPMAS transfer dynamics becomes

$$
\begin{gathered}
H_{I-S}^{C P}(t)=-\omega_{1 I} I_{x}+2 b(t) I_{z} S_{z}^{2-3}+6 b(t) I_{z} S_{z}^{1-4}+ \\
\left\{\Omega_{S}^{\prime}(t)+\Omega_{Q, S}^{2-3}(t)\right\}\left[\cos ^{2} \frac{\theta}{2} S_{z}^{2-3}+\left\{2 \sin \theta S_{y}^{1-2}-\sin \frac{\theta}{2} S_{y}^{3-4}\right\} \cos \int_{0}^{t} \omega_{e}\left(t^{\prime}\right) d t^{\prime}\right. \\
\left.-\left\{2 \sin \theta S_{x}^{1-2}-\sin \frac{\theta}{2} S_{x}^{3-4}\right\} \sin \int_{0}^{t} \omega_{e}\left(t^{\prime}\right) d t^{\prime}\right]+ \\
\left\{3 \Omega_{S}^{\prime}(t)+\Omega_{Q, S}^{1-4}(t)\right\}\left[\cos ^{2} \frac{\theta}{2} S_{z}^{1-4}+\left\{2 \sin \theta S_{y}^{1-2}-\sin \frac{\theta}{2} S_{y}^{3-4}\right\} \cos \int_{0}^{t} \omega_{e}\left(t^{\prime}\right) d t^{\prime}\right. \\
\left.-\left\{2 \sin \theta S_{x}^{1-2}-\sin \frac{\theta}{2} S_{x}^{3-4}\right\} \sin \int_{0}^{t} \omega_{e}\left(t^{\prime}\right) d t^{\prime}\right]+ \\
2 \omega_{1 S} A(t)\left[\cos ^{2} \frac{\theta}{2} S_{x}^{2-3}-\sin ^{2} \frac{\theta}{2} S_{x}^{1-4}+2 \sin \theta S_{x}^{1-3}-2 \sin \theta S_{x}^{2-4}\right],
\end{gathered}
$$

where $\omega_{1 I}=\gamma_{I} B_{1 I}$ represents the on-resonance irradiation of the $I$-spin, $\Omega_{S}^{\prime}(t)=\Omega_{S}+$ $\Delta \omega / 2-\left(\Delta \omega / t_{p}\right) t$, and the MAS-modulated heteronuclear dipolar coupling of the I-S pair is given by

$$
b(t)=\sum_{k=-2, k \neq 0}^{2} b_{k} e^{i k \omega_{r} t} .
$$

The orientation-dependent $\left\{b_{k}\right\}$ coefficients in Eq. (23) correlate an I-S dipolar vector defined in its principal axis system to the rotor frame, ${ }^{15}$ as related by the Euler angles $\beta_{d}$ and $\gamma_{d}$. Any 
potential homonuclear $I-I$ and $S$-S dipolar coupling interactions were disregarded, and $I$ - and S-spin CSAs were again ignored for simplicity.

The Hamiltonian in Eq. (22) can be used to consider both the $I \rightarrow S(\mathrm{CT})$ and the $I \rightarrow S$ (TQ) BRAIN-CPMAS dynamics, described by the $S_{z}^{2-3} \& S_{x}^{2-3}$ and $S_{z}^{1-4} \& S_{x}^{1-4}$ operators, respectively. These two dynamic processes, however, are not decoupled from one another: they are be coupled by two pairs of operators, $S_{x}^{1-3}$ and $S_{x}^{2-4}$, and $S_{\xi}^{1-2}$ and $S_{\xi}^{3-4}$ $(\xi=x$ or $y$ ), arising from the tilt transformation and quadrupolar modulation (cf. Eq. (22)). The fact that the rf Hamiltonian still contains a $S_{x}^{2-3}$ operator in the case of $\theta \approx 0$, however, means that the CT can be effectively spin-locked with a smaller $v_{1 S}$ than the TQ transition, making the $I \rightarrow S(\mathrm{CT})$ process more favorable than the $I \rightarrow S(\mathrm{TQ})$ one. The efficiency of this spin-locking behavior can be evaluated in terms of the adiabaticity parameter, $\alpha=\frac{v_{1 S}^{2}}{C_{Q} v_{r}}$, which specifies the extent of rf-induced level-crossings. ${ }^{19}$ When $\alpha \ll 1$, the system is said to be in the sudden passage regime; under these conditions, the rf does not promote crossings among different energy levels. As discussed within the context of Fig. 2, a $v_{1 S}$ value in the range of 3 to $10 \mathrm{kHz}$, along with a high spinning rate ( $\left.v_{r} \geq 20 \mathrm{kHz}\right)$, places a prototypical $S=$ 3/2 nucleus with a $C_{\mathrm{Q}}$ magnitude ranging from ca. 1 to $10 \mathrm{MHz}$ under optimal spin-locking conditions $\left(\omega_{Q} \gg \omega_{1 S} ; \theta \approx 0 ; \omega_{e} \approx \omega_{Q}\right)$. Under these circumstances, the oscillating terms can be dropped from the rf Hamiltonian, and thereby the TQC and the oscillatory terms that connect the CTC to other coherences in Eq. (22) can be safely ignored. Focusing on a CP process within the effective, relevant $S_{\xi}^{2-3}$ subspace, Eq. (22) simplifies to:

$$
H_{I-S}^{C P}(t)=-\omega_{1 I} I_{x}+\left\{\Omega_{S}^{\prime}(t)+\Omega_{Q, S}^{2-3}(t)+2 b(t) I_{z}\right\} S_{z}^{2-3}+2 \omega_{1 S} S_{x}^{2-3},
$$


where for convenience we assumed $A(t)=1$. This description of BRAIN-CPMAS can in fact be extended to higher half-integer quadrupolar nuclei $(S=3 / 2,5 / 2,7 / 2$, or $9 / 2$ ), by defining $S_{z}=S_{z}^{c-t}$ and $S_{x}=\left(S+\frac{1}{2}\right) S_{x}^{c-t}$, where $c$-t stands for 2-3, 3-4, 4-5, and 5-6 for $S=3 / 2,5 / 2$, 7/2, and 9/2, respectively. Using these fictitious spin-1/2 operators, Eq. (24) then becomes

$$
H_{I-S}^{C P}(t)=-\omega_{1 I} I_{x}+\left\{\Omega_{S}^{\prime}(t)+\Omega_{Q, S}^{c-t}(t)\right\} S_{z}+\omega_{1 S} S_{x}+2 b(t) I_{z} S_{z}
$$

Except for an additional $\Omega_{Q, S}^{c-t}(t)$ second-order term that influences the effective offset, Eq. (25) is identical to $I(1 / 2) \rightarrow S(1 / 2)$ BRAIN-CPMAS equations that we developed previously. ${ }^{24}$ The expression of $\Omega_{Q, S}^{c-t}(t)$ for an arbitrary half-integer quadrupolar spin $S$ is

$$
\Omega_{Q, S}^{c-t}(t)=\frac{3}{\omega_{0}} \chi_{Q}^{2}[4 S(S+1)-3] \times \sum_{m=-4}^{4} \omega_{Q}^{m}(\alpha, \beta) \exp \left[i m\left(\gamma+\omega_{r} t\right)\right]
$$

where explicit expressions for $\omega_{Q}^{m}(\alpha, \beta)$ are summarized in the Appendix.

With these expressions, a closed analytical form can be found for the Hamiltonian governing BRAIN-CPMAS in the central transitions of half-integer quadrupoles. To do so, we apply a rotational transformation on Eq. (26) into a doubly tilted frame, ${ }^{15,24}$ where all of the spin-locked magnetizations of interest lie parallel to the effective fields in this frame:

$$
\begin{aligned}
\widetilde{H}_{I-S}^{C P}(t) & =-\omega_{e I} I_{z}-\omega_{e S}(t) S_{z}+2 \sin \theta_{I} \sin \theta_{S}(t) b(t) I_{x} S_{x}+2 \cos \theta_{I} \cos \theta_{S}(t) b(t) I_{z} S_{z} \\
& -2 \cos \theta_{I} \sin \theta_{S}(t) b(t) I_{z} S_{x}-2 \sin \theta_{I} \cos \theta_{S}(t) b(t) I_{x} S_{z} .
\end{aligned}
$$

The effective resonance frequencies along this doubly tilted frame are

$$
\begin{gathered}
\omega_{e I}=\omega_{1 I} \\
\omega_{e S}(t)=\sqrt{\left\{\Omega_{S}+\Omega_{Q, S}^{c-t}(t)+\frac{\Delta \omega}{2}-\frac{\Delta \omega}{t_{p}} t\right\}^{2}+\omega_{1 S}^{2}}
\end{gathered}
$$

while the tilt angles $\theta$ and $\theta_{\mathrm{s}}(\mathrm{t})$ that relate the $z^{\prime}$-axes to the $z$-axes in the previous frame are: 


$$
\begin{gathered}
\cos \left(\theta_{I}\right)=\Omega_{I} / \omega_{e I}=0 ; \sin \left(\theta_{I}\right)=\omega_{1 I} / \omega_{e I}=1 \\
\cos \left(\theta_{S}[t]\right)=\left(\Omega_{S}+\Omega_{Q, S}^{c-t}(t)+\frac{\Delta \omega}{2}-\frac{\Delta \omega}{t_{p}} t\right) / \omega_{e S}(t) \\
\sin \left(\theta_{S}[t]\right)=\omega_{1 S} / \omega_{e S}(t) .
\end{gathered}
$$

With these definitions and assumptions, Eq. (27) is reduced to:

$$
\begin{gathered}
\widetilde{H}_{T}^{\prime}(t)=-\omega_{1 I} I_{z}-\omega_{e S}(t) S_{z}+2 \sin \theta_{S}(t) b(t) I_{x} S_{x}= \\
-\omega_{1 I} I_{z}-\omega_{e S}(t) S_{z}+\frac{2 \omega_{1 S} b(t)}{\omega_{e S}(t)} I_{x} S_{x}
\end{gathered}
$$

Although the $\omega_{e s}(t)$ term is complicated due to the presence of the second-order quadrupolar oscillation, Eq. (33) still closely resembles the usual Hamiltonian describing the HH transfer for $I=S=1 / 2 .^{14,15,17}$ Utilizing the single-transition operator formalism normally used to analyze these transfers, Eq. (33) can be rewritten as:

$$
\widetilde{H}_{T}^{\prime}(t)=-\omega_{\Delta}(t) I_{z}^{Z Q}-\omega_{\Sigma}(t) I_{z}^{D Q}+\frac{\omega_{1 S}\left[b_{ \pm 1} e^{ \pm i \omega_{r} t}+b_{ \pm 2} e^{ \pm 2 i \omega_{r} t}\right]}{\omega_{e S}(t)}\left(I_{x}^{Z Q}+I_{x}^{D Q}\right),
$$

where $\omega_{\Delta}(t)=\omega_{1 I}-\omega_{e S}(t), \omega_{\Sigma}(t)=\omega_{1 I}+\omega_{e S}(t)$, and the single-transition operators for the zero-quantum (ZQ) and double-quantum (DQ) coherences are defined by:

$$
\begin{array}{cc}
I_{z}^{Z Q}=\frac{I_{z}-S_{z}}{2}, & I_{z}^{D Q}=\frac{I_{z}+S_{z}}{2} \\
I_{x}^{Z Q}=\frac{I_{+} S_{-}+I_{-} S_{+}}{2}, & I_{x}^{D Q}=\frac{I_{+} S_{+}+I_{-} S_{-}}{2} \\
I_{y}^{Z Q}=\frac{I_{+} S_{-}-I_{-} S_{+}}{2 i}, & I_{y}^{D Q}=\frac{I_{+} S_{+}-I_{-} S_{-}}{2 i} .
\end{array}
$$

As the single transition operators defined in these ZQ and DQ subspaces commute with each other, their time evolutions can be solved separately 


$$
\widetilde{H}_{T}^{Z Q}(t)=-\omega_{\Delta}(t) I_{Z}^{Z Q}+\frac{\omega_{1 S}\left[b_{ \pm 1} e^{ \pm i \omega_{r} t}+b_{ \pm 2} e^{ \pm 2 i \omega_{r} t}\right]}{\omega_{e S}(t)} I_{x}^{Z Q}
$$

and

$$
\widetilde{H}_{T}^{D Q}(t)=-\omega_{\Sigma}(t) I_{z}^{D Q}+\frac{\omega_{1 S}\left[b_{ \pm 1} e^{ \pm i \omega_{r} t}+b_{ \pm 2} e^{ \pm 2 i \omega_{r} t}\right]}{\omega_{e S}(t)} I_{x}^{D Q}
$$

These equations are identical to forms that we have recently derived for $I=S=1 / 2$ cases. ${ }^{24}$ We can therefore use our previous analysis to consider the evolutions that they will impart. Under the fast spinning and slow adiabatic sweep conditions that are of interest in this study, we have shown that the average Hamiltonian over the time scale of a rotor period may be approximated by assuming a quasi-constant offset value $\omega_{\mathrm{p}}\left(t_{0}\right)$. Thus, an average Hamiltonian over a rotor period $\left\langle H_{T}^{\zeta}\right\rangle_{a v}=\frac{1}{\tau_{r}} \int_{0}^{\tau_{r}} H_{T}^{\zeta}\left(t^{\prime}\right) d t^{\prime}, \zeta=$ ZQ or DQ, can be obtained from Eqs. (36) and (37) via the transformation $U_{p} H_{T}^{\zeta} U_{p}^{-1}-i U_{p} \frac{d}{d t} U_{p}^{-1}$, where $U_{p}=$ $\exp \left(i k \omega_{r} t I_{z}^{\zeta}\right)$ and $\zeta=\mathrm{ZQ}$ or DQ refers to the zero- or double-quantum sub-spaces. This produces

$$
\left\langle H_{T}^{\zeta}\right\rangle_{a v}=\left[-\left(\omega_{1 \mathrm{I}}-k \omega_{r}\right)+m \omega_{e S}(t)\right] I_{z}^{\zeta}+\frac{\omega_{1 S} b_{k}}{2 \omega_{e S}(t)} I_{+}^{\zeta},
$$

where $k= \pm 1$ and $\pm 2, I_{+}^{\zeta}=I_{x}^{\zeta}+i I_{y}^{\zeta}$, and $m$ is +1 for the ZQ-CP process or -1 for the DQCP process.

\subsection{Hartmann-Hahn matching conditions in BRAIN CPMAS: The influence of the second-order quadrupolar effects}

As in the $I=S=1 / 2$ case, time-progressive ZQ- and DQ-HH matching conditions can affect the $I(1 / 2) \rightarrow S(\mathrm{CT} ; S \geq 3 / 2)$ dynamics over the course of the frequency swept pulse. A 
difference however, now stems from the presence of the second-order quadrupolar terms in the effective offset frequency $v_{e S}(t)=\omega_{e S}(t) / 2 \pi$, that participates in defining these $\mathrm{HH}$ matching conditions. Moreover, because of the MAS-averaged second-order quadrupolar effects, ${ }^{60}$ the ZQ- and DQ-matching conditions can evidence powder angle dependencies depending on the quadrupolar tensor. This section inspects the time-progressive conditions that set the $I_{z}^{D Q}$ or $I_{z}^{Z Q}$ coefficients in Eq. (38) equal to zero for these ZQ and DQ processes, during $I(1 / 2) \rightarrow S(C T ; S \geq 3 / 2)$ BRAIN-CPMAS.

As these conditions are satisfied, I-spin polarization will be transferred to the CTC of the $S$-spin. $\mathrm{A} \mathrm{ZQ}_{ \pm k}(k=1$ or 2$) \mathrm{HH}$ matching condition is satisfied when the effective frequency, $v_{e S}(t)$ - which depends on $v_{1 S}, \Omega_{s}, \omega_{p}(t)$, and the orientation-dependent secondorder quadrupolar frequency $\Omega_{Q, S}^{c-t}(t)$ - instantaneously satisfies $v_{1 I}-v_{e S}(t)= \pm k v_{r}$. In a similar manner, a $\mathrm{DQ}_{k}(k=1$ or 2$) \mathrm{HH}$ matching condition is met when $v_{e S}(t)$ satisfies $v_{1 I}+v_{e S}(t)=k v_{r}$. Figure 3 shows numerical simulations of $I(1 / 2) \rightarrow S(\mathrm{CT} ; S=3 / 2)$ BRAIN-CPMAS profiles deriving from these considerations. These simulations incorporate parameters for an isolated I-S pair that are taken from ${ }^{1} \mathrm{H}-{ }^{11} \mathrm{~B}$ BRAIN-CPMAS experiments on sodium tetraborate decahydrate (vide infra), assuming a relatively fast MAS rate $\left(v_{r}=25\right.$ $\mathrm{kHz}$ ). Figure 3a shows the ZQ- and DQ-HH conditions revealed as a function of the $I$-spin rf strength for a fixed $v_{1 S}(=8 \mathrm{kHz})$. Because of the orientation-dependent quadrupolar frequency dispersion in $v_{e S}(t)$, numerous local maxima and minima showing positive and negative signal enhancements of the CTC, arise. Still, the $\mathrm{ZQ}_{1}, \mathrm{ZQ}_{2}, \mathrm{DQ}_{1}$ and $\mathrm{DQ}_{2}$ conditions are all clearly visible in this rf field profile, with the signal enhancements from ZQ- and DQHH matching conditions possessing the expected opposite signs. Also illustrated in Fig. 3b3e are the time-dependent transfer dynamics revealed by BRAIN-CPMAS simulations, for 
the (b) $\mathrm{DQ}_{1}$, (c) $\mathrm{DQ}_{2}$, (d) $\mathrm{ZQ}_{1}$ and (e) $\mathrm{ZQ}_{2}$ conditions found in Fig. 3a. In these cases, the spin-locked $\left(S_{z}\right)$ and transverse $\left(S_{x}\right)$ states are null at the beginning of the swept pulse, while all polarization sits at $I_{\mathrm{z}}$. As the offset of the $S$-spin rf changes over the duration of the swept pulse, the magnetization of the spin-locked state grows gradually, via HH transfers associated with the $\mathrm{DQ}_{ \pm k}$ and $\mathrm{ZQ}_{ \pm k}(k=0,1$ or 2$)$ conditions.

While similar mechanisms are active in BRAIN-CPMAS between spin-1/2 nuclides, there are a number of peculiarities in the dynamics shown in Fig. 3 as compared to the previously reported $S=1 / 2$ case. ${ }^{24}$ These include (1) rf scaling effects coming from the coefficient 2 in front of the $S_{x}^{1 / 2}$ operator; (2) the absence of sharp $S_{z}$ or $S_{x}$ buildup points associated with the fulfillment of $v_{e S}(t) \pm v_{e I}-k v_{r}=0(k=1$ or 2$)$ conditions throughout the powdered sample; (3) the fact that polarization accrued by the S-spin during the BRAINCP process stays mainly as a longitudinal magnetization $S_{z}$ without developing any significant transverse components, $S_{x}$ and $S_{y}$ (only $S_{x}$ is shown in the figure); and 4) the fact that the CP-enhanced $S_{z}$ magnetization does not oscillate back to $I_{z}$ (at least to some extent) during the CP mixing period, even though a single I-S pair is being considered. The last three of these features can be attributed to the presence of a distribution of second-order quadrupolar anisotropic frequencies, $\Omega_{Q, S}^{c-t}(t)$, in $v_{e S}(t)$. Because of these anisotropic shifts, the $v_{e S}(t) \pm v_{e I}-k v_{r}=0$ conditions are met at different times over the course of the swept pulse for different crystal orientations, resulting in a spread of the $\mathrm{HH}$ transfer processes throughout the $0-t_{p}$ time period. Consequently, one does not observe any specific time points that are explicitly associated with ZQ or DQ conditions. This "spreading effect" associated with $\Omega_{Q, S}^{c-t}$ also "washes out" the oscillating features of $S_{z}$ magnetization transferred during the course of the BRAIN-CPMAS process, which therefore never returns back to $I_{z}$ to any 
appreciable extent. Interestingly, the presence of the $\Omega_{Q, S}^{c-t}(t)$ term in $v_{e S}(t)$ also obscures another phenomenon that was clearly evident in the $I\left({ }^{1} \mathrm{H}\right) \rightarrow S(1 / 2)$ case, and even in the $I\left({ }^{1} \mathrm{H}\right) \rightarrow S(S=1)$ case: ${ }^{24,43}$ the occurrence of rotary resonance (RR) inversion effects. Discussed in detail in Ref. 24, these phenomena originated in rotor-driven anisotropic oscillations of either the CSA or the first-order quadrupolar interactions (for $S=1$ ), whenever the spin-locked magnetization passed through effective field conditions satisfying $v_{e S}(t)=$ $k v_{r}{ }^{64,65}$ Once again, second-order broadenings spread out this condition, implying that there will exist no singular time point over the course the adiabatic frequency sweep, where the spin-locked S-spin polarization will be suddenly inverted due to a RR condition.

Figure 3 illustrates various aspects of the BRAIN-CPMAS transfer dynamics for a sweep bandwidth $\Delta v<v_{r}$. Although no noticeable sidebands arise on either side of the isotropic central transition spectra for this set of MAS rates and $C_{Q}$ values, calculations (not shown) indicate that $\Delta v$ values that exceed $v_{r}$ completely change the characteristics of these curves. This is due to the interfering effects that arise whenever an rf sweep affects both CT and ST sideband manifolds, as illustrated in Figure 1. This leads to an inefficient spinlocking of the polarization being accrued along $S_{z}$, and thereby to a loss of CP efficiency. It follows that for efficient $\mathrm{CP}$, the width of $\Delta v$ in these experiments should be set to exceed the bandwidth of the second-order quadrupolar anisotropic frequency exhibited by the CT under MAS, but kept below the spinning rate. This is another major difference with respect to the BRAIN-CPMAS experiments we had discussed for $S=1 / 2$ and 1 cases.

\section{Materials and Methods}

\subsection{Numerical calculations}


All simulations were carried out in the time-domain with full Hamiltonians (Eqs. [1]-[6] and Eq. [16]), without any approximations other than for a rotating frame transformation. These calculations utilized in-house simulation programs written in Matlab ${ }^{\circledR}$ (The Mathworks Inc.). Time evolutions were evaluated numerically by propagating density matrices using piecewise $(2 \mu \mathrm{s})$ increments, which were chosen to take into account the variations of the amplitudes and phases of the rf waveforms and the MAS-driven rotational modulations of the quadrupolar and dipolar interactions (CSAs were ignored for simplicity). An isolated $S=3 / 2$ site was considered for examining the inversion and level-crossing effects, and an isolated $I=$ 1/2, $S=3 / 2$ spin pair with a suitable MAS-modulated dipolar Hamiltonian (dipolar coupling constant $=8 \mathrm{kHz}$ ) were used for examining the $\mathrm{DQ}_{ \pm k}$ and $\mathrm{ZQ}_{ \pm k}(k=0,1$ or 2$) \mathrm{HH}$ signal transfers under MAS. Powder averages were accounted for in the calculations by considering 538 different crystal orientations subtending the ZCW Euler angle set, ${ }^{66}$ assuming coincident and axially symmetric dipolar and quadrupolar tensor orientations for simplicity.

\subsection{Experimental}

${ }^{1} \mathrm{H}-{ }^{23} \mathrm{Na}$ and ${ }^{1} \mathrm{H}_{-}{ }^{11} \mathrm{~B}$ ramped CPMAS and BRAIN-CPMAS experiments were carried out on powdered samples of sodium tetraborate decahydrate and sodium citrate dihydrate. Samples were purchased from Sigma-Aldrich (St. Louis, MO) and used without further treatment. About $15 \mathrm{mg}$ of each sample were packed into a $2.5 \mathrm{~mm}$ MAS Bruker rotor and spun at $25 \mathrm{kHz}$. All experiments were carried out at room temperature in an $11.75 \mathrm{~T}$ magnet

equipped with a Bruker Avance ${ }^{\circledR}$ console operating at ${ }^{1} \mathrm{H},{ }^{23} \mathrm{Na}$ and ${ }^{11} \mathrm{~B}$ frequencies of 500.92, 150 and $164.1 \mathrm{MHz}$, respectively. The swept rf pulse shapes were constructed utilizing the shaped pulse tool of the Bruker Topspin ${ }^{\circledR}$ software; these pulses utilized a WURST 
amplitude profile $v_{1 s}\left(1-\cos ^{40}\left[\pi t / \tau_{p}\right]\right)$ and ramped phases corresponding to a linear frequency sweep. 2000 data points were used to digitize these pulse shapes, with sweep bandwidths of $5 \leq \Delta v \leq 40 \mathrm{kHz}$ and pulse lengths of $2 \leq t_{p} \leq 14 \mathrm{~ms}$. For $v_{r}=25 \mathrm{kHz}$, optimal BRAIN-CPMAS matching parameters were found for $\Delta v=10 \mathrm{kHz}$ and $t_{p}=8 \mathrm{~ms}$. Optimal ${ }^{23} \mathrm{Na} /{ }^{11} \mathrm{~B}$ and ${ }^{1} \mathrm{H}$ rf power conditions were then sought experimentally by sweeping both rf channel intensities independently. As predicted by Eq. (32), these parameters were usually found to satisfy the $\mathrm{DQ}_{1}$ and $\mathrm{ZQ}_{1}$ conditions $v_{e S}=v_{r} \pm v_{1 I}$ (where $v_{e S}$ includes the MAS-modulated second-order quadrupolar interaction and instantaneous offset frequency arising from the adiabatic sweep). For comparison, conventional CPMAS NMR experiments were also carried out with independent rf pulse power optimizations, employing a ramped (90\%-110\%) spin-lock pulse on the ${ }^{1} \mathrm{H}$ channel while simultaneously applying a rectangular spin-lock pulse on the S-channel. ${ }^{11,67,68}$ The mixing time used in these CPMAS experiments was $0.5 \sim 2 \mathrm{~ms}$, based on optimizations. All NMR spectra were acquired by co-adding 128 transients with recycle delays of ca. 4 s. $90^{\circ}{ }^{1} \mathrm{H}$ and quadrupolar pulses were $2 \mu$ s for all experiments. SPINAL-64 ${ }^{69}{ }^{1} \mathrm{H}$ decoupling was used during the acquisition period, with a $100 \mathrm{kHz}$ decoupling rf field.

\section{Results}

We investigated the performance of ${ }^{1} \mathrm{H}-{ }^{11} \mathrm{~B}$ BRAIN-CPMAS for enhancing the NMR signals in the ${ }^{11} \mathrm{~B}$ NMR spectra under fast spinning conditions. As a first step, we explored optimal sets of $v_{1 I}$ and $v_{1 S}$ strengths experimentally under a fixed set of $\Delta v, t_{p}$, and $v_{r}$ parameters. Figure 4 shows the optimal $v_{1 I}\left({ }^{1} \mathrm{H}\right)$ and $v_{1 S}\left({ }^{11} \mathrm{~B}\right)$ rf field strengths determined for BRAIN ( $a$ and b) and ramped HH-CPMAS (c and d), using sodium tetraborate decahydrate 
as a model compound. For the BRAIN-CPMAS case, an optimal ${ }^{11} \mathrm{~B}$ rf field was found at $5 \pm 3 \mathrm{kHz}$ (Fig. 4b) when $v_{1 I}\left({ }^{1} \mathrm{H}\right)$ was set to $55 \pm 2 \mathrm{kHz}$, with $\Delta v=25 \mathrm{kHz}$ and $v_{r}=31 \mathrm{kHz}$. (this corresponds to the $\mathrm{DQ}_{2}$ condition). Other modes visible in the $v_{1 I}\left({ }^{1} \mathrm{H}\right)$ sweep profiles, such as $\mathrm{DQ}_{1}\left(v_{1 I}=26 \pm 3 \mathrm{kHz} ; v_{1 S}=5 \pm 3 \mathrm{kHz}\right), \mathrm{ZQ}_{1}\left(v_{1 I}=35 \pm 3 \mathrm{kHz} ; v_{1 S}=5 \pm 3 \mathrm{kHz}\right)$ and $\mathrm{ZQ}_{2}\left(v_{1 I}=65 \pm 3 \mathrm{kHz} ; v_{1 S}=5 \pm 3 \mathrm{kHz}\right.$ ), were not as efficient as the $\mathrm{DQ}_{2}$ mode (Figs. 4a, 4b). For HH-CPMAS using a 90\%-110\% ramp pulse on the ${ }^{1} \mathrm{H}$ channel, optimized $v_{1 S}\left({ }^{11} \mathrm{~B}\right)$ and $v_{1 I}\left({ }^{1} \mathrm{H}\right)$ rf fields were found at the $\mathrm{ZQ}_{2}\left(v_{1 S}=14 \pm 4 \mathrm{kHz} ; v_{1 I}=75 \pm 2 \mathrm{kHz}\right)$ and the $\mathrm{ZQ}_{1}\left(v_{1 S}=14 \pm 4 \mathrm{kHz} ; v_{1 I}=49 \pm 3 \mathrm{kHz}\right.$ ) conditions. Additional relevant $\mathrm{CP}$ conditions for the ramped HH-CPMAS case are indicated in Fig. 4c and 4d.

Figure 5 compares BRAIN-CPMAS, ramped HH-CPMAS and directly-excited ${ }^{11} \mathrm{~B}$ MAS spectra of sodium tetraborate decahydrate. Both BRAIN-CPMAS and HH-CPMAS spectra were acquired after optimizing $v_{1 S}\left({ }^{11} \mathrm{~B}\right)$ and $v_{1 I}\left({ }^{1} \mathrm{H}\right)$, according to data shown in Fig. 4. Red numbers are shown next to each spectrum, representing the relative peak intensities of the site with the smaller $\mathrm{C}_{\mathrm{Q}}$ value $\left(1.8 \mathrm{MHz}, \eta_{Q}=0.6\right)$ in comparison to this peak intensity in the direct-excitation spectrum, which is set to 100 , and used to normalize all other peak intensities. Only the spectra from the most efficient modes of broadband CP are included in this comparison: $\mathrm{DQ}_{2}$ for BRAIN-CPMAS and $\mathrm{ZQ}_{2}$ for HH-CPMAS. Also included on the top two rows are simulations of the idealized quadrupolar patterns based on the known parameters from the literature $\left(C_{\mathrm{Q}}=1.8 \mathrm{MHz}, \eta_{\mathrm{Q}}=0.6 ; \mathrm{C}_{\mathrm{Q}}=7.8 \mathrm{MHz}, \eta_{\mathrm{Q}}=\right.$ 0.1). A good match between experiment and simulation arises from the BRAIN-CPMAS spectrum, which has lower $\mathrm{S} / \mathrm{N}$ but fewer distortions than its ramped HH-CPMAS counterpart. Also note that the ${ }^{1} \mathrm{H}$ and ${ }^{11} \mathrm{~B}$ rf pulse strengths are weakest for this BRAINCPMAS spectrum $\left(v_{11}[1 \mathrm{H}]=55 \mathrm{kHz} ; v_{1 S}[11 \mathrm{~B}]=5 \mathrm{kHz}\right)$. 
Figure 6 shows the ${ }^{1} \mathrm{H}_{-}{ }^{23} \mathrm{Na}$ BRAIN-CPMAS spectra of (a) sodium tetraborate decahydrate and (b) sodium citrate dihydrate, acquired after individual optimizations of both of the $v_{1 I}$ and $v_{1 S}$ rf fields. Each BRAIN-CPMAS spectrum is compared to the corresponding HH-CPMAS spectrum, both obtained after individual optimization; also included are direct-excitation spectra acquired using a single 90-degree pulse that was also optimized. The relative signal gains of the BRAIN- and HH-CPMAS experiments are comparable. Furthermore, both BRAIN-CPMAS and HH-CPMAS spectra of sodium tetraborate decahydrate possess higher signal intensities than their directly polarized counterpart, in accordance with the observations by Harris and Nesbitt. ${ }^{70}$

\section{Discussion and Conclusions}

In static solids, BRAIN-CP possesses distinct advantages over conventional $\mathrm{CP}$ in terms of widening the effective bandwidth of the polarization transfer. ${ }^{31-33}$ MAS considerably complicates the workings of this method, ${ }^{24}$ yet we found that from a practical perspective its applications on $I(1 / 2) \rightarrow S(1 / 2)$ polarization transfers are still useful, particularly when fast spinning rates $(\geq 25 \mathrm{kHz})$ were employed. ${ }^{24}$ In the current work, the BRAIN-CPMAS method was extended to half-integer quadrupolar nuclei. From the standpoint of spin physics this extension complicates matters even further, owing to quadrupole-driven level crossings that disrupt the spin-lock of the transition one is attempting to polarize. The use of an rf sweep further complicates matters, as such rf will affect multiple central and satellite sideband manifolds, thereby also conspiring against the spin-locking efficiency (Fig. 1). Thus, by contrast to BRAIN-CPMAS in $S=1 / 2$ and 1 cases, where the sweep range is

limited by the MAS rate,, ${ }^{24,52}$ in half-integer quadrupolar BRAIN-CPMAS the swept pulse 
parameters must be optimized according to the magnitude of both $C_{Q}$ and the spinning rate $v_{r}$. Under these conditions, the optimal pulse power for BRAIN-CPMAS, $v_{1 S}$, ends up having a smaller magnitude than its HH counterpart (Figure 4). At these small and easily achievable $v_{1 S}$ values, the quadrupolar-driven level crossing effects associated with spin-locking a central $+1 / 2 \leftrightarrow-1 / 2$ transition, ${ }^{43,49}$ are minimized (Figure 2). Moreover, by using small $v_{1 S}$, nearly ideal spectral lineshapes are obtained for the central transition spectrum. The method is thus expected to operate best at high magnetic fields, where second-order quadrupolar effects are scaled down and quadrupolar-induced complications are minimized.

Notwithstanding these considerations, we observe that the experimental BRAINCPMAS spectra for half-integer quadrupolar nuclei may often have lower $\mathrm{S} / \mathrm{N}$ than their directly excited counterparts. Although comparison of $\mathrm{S} / \mathrm{N}$ in direct excitation and $\mathrm{CP}$ experiments was not exhaustively investigated herein, we ascribe the diminished $\mathrm{S} / \mathrm{N}$ in the instances where it was observed (Figs. 5 and $6 \mathrm{~b}$ ) to the absence of strong $I\left({ }^{1} \mathrm{H}\right)-S$ dipolar interactions. Other interesting systems, containing for instance directly-bonded ${ }^{1} \mathrm{H}-{ }^{17} \mathrm{O}$ spin pairs, might be more amenable for study by BRAIN-CPMAS methods. Moreover, the advent of high-field dynamic nuclear polarization (DNP) has introduced new opportunities for NMR of heteronuclei. ${ }^{71-77}$ The transfer of electronic polarization to protons, and subsequent broadband CP transfer to half-integer quadrupoles, ${ }^{78}$ would certainly benefit from methodologies like BRAIN, which could thereby open up new avenues for applications of quadrupolar NMR to a wide array of materials.

Acknowledgments. This work was supported by the NHMFL through the National Science Foundation Cooperative Agreement (DMR-0084173) and by the State of Florida. L.F. 
acknowledges support from the Israel Science Foundation grant 795/13, the Kimmel Institute for Magnetic Resonance (Weizmann Institute), and the generosity of the Perlman Family Foundation. R.W.S. thanks NSERC for funding this research in the form of a Discovery Grant and Discovery Accelerator Supplement, and is also grateful for an Early Researcher Award from the Ontario Ministry of Research and Innovation, and for a $50^{\text {th }}$ Anniversary Golden Jubilee Chair from the University of Windsor.

\section{Appendix}

The explicit expressions of $\omega_{Q}(t)$ and $\Omega_{Q, S}^{c-t}(t)$ terms are provided according to the tensor transformations:

$$
\operatorname{EFG}(S) \stackrel{\{\alpha, \beta, \gamma\}}{\longrightarrow} \text { ROTOR } \stackrel{\left\{\omega_{r} t, 54.7^{\circ}, 0^{\circ}\right\}}{\longrightarrow} L A B
$$

and provided as:

$$
\begin{array}{r}
\omega_{Q}(t)=\frac{3}{2 S(2 S-1)}\left(\frac{e^{2} q Q}{\hbar}\right)\left[C_{1} \cos \left(\omega_{r} t+\gamma\right)+C_{2} \cos \left(2 \omega_{r} t+2 \gamma\right)+\right. \\
\left.S_{1} \sin \left(\omega_{r} t+\gamma\right)+S_{2} \sin \left(2 \omega_{r} t+2 \gamma\right)\right]
\end{array}
$$

where

$$
\begin{gathered}
C_{1}=-\frac{1}{\sqrt{2}} \sin 2 \beta\left(1-\frac{\eta_{Q}}{3} \cos 2 \alpha\right) \\
C_{2}=\frac{1}{2}\left[\sin ^{2} \beta+\frac{\eta_{Q}}{3}\left(\cos ^{2} \beta+1\right) \cos 2 \alpha\right] \\
S_{1}=-\frac{\sqrt{2}}{3} \eta_{Q} \sin \beta \sin 2 \alpha \\
S_{2}=-\frac{1}{3} \eta_{Q} \cos \beta \sin 2 \alpha
\end{gathered}
$$




$$
\Omega_{Q, S}^{c-t}(t)=\frac{3}{\omega_{0}}\left[\frac{e^{2} q Q / \hbar}{2 S(2 S-1)}\right]^{2}[4 S(S+1)-3] \times \sum_{m=-4}^{4} \omega_{Q}^{m}(\alpha, \beta) \exp \left[\operatorname{im}\left(\gamma+\omega_{r} t\right)\right]
$$

where

$$
\begin{gathered}
\omega_{Q}^{0}(\alpha, \beta)=\frac{5}{96} A^{2}+\frac{1}{3}\left(\frac{B^{2}}{4}+\frac{C^{2}}{9}\right)+\frac{23}{48}\left(\frac{D^{2}}{4}+\frac{E^{2}}{9}\right) \\
\omega_{Q}^{ \pm 1}(\alpha, \beta)=\frac{1}{24 \sqrt{2}}\left(\frac{B D}{4}-\frac{A B}{2}-\frac{C E}{9} \pm \frac{i}{6}[B E+C D-6 A C]\right) \\
\omega_{Q}^{ \pm 2}(\alpha, \beta)=\frac{1}{48}\left(\frac{4 C^{2}}{9}-A D-B^{2} \pm \frac{2 i}{3}[2 B C-A E]\right) \\
\omega_{Q}^{ \pm 3}(\alpha, \beta)=\frac{7}{24 \sqrt{2}}\left(\frac{B D}{4}+\frac{C E}{9} \pm \frac{i}{6}[B E-C D]\right) \\
\omega_{Q}^{ \pm 4}(\alpha, \beta)=-\frac{7}{96}\left(\frac{D^{2}}{9}-\frac{E^{2}}{9} \pm \frac{i}{3} D E\right),
\end{gathered}
$$

and

$$
\begin{gathered}
A=3 \cos ^{2} \beta-1+\eta_{Q} \sin ^{2} \beta \cos 2 \alpha \\
B=\sin ^{2} \beta\left(1-\frac{\eta_{Q}}{3} \cos 2 \alpha\right) \\
C=\eta_{Q} \sin \beta \sin 2 \alpha \\
D=\sin ^{2} \beta+\frac{\eta_{Q}}{3}\left(\cos ^{2} \beta+1\right) \cos 2 \alpha \\
E=\eta_{Q} \cos \beta \sin 2 \alpha .
\end{gathered}
$$

For $\mathrm{S}=3 / 2$ and $\eta_{Q}=0$ case it becomes further simplified into:

$$
\Omega_{Q, S}^{2-3}(t)=\frac{1}{\omega_{0}}\left[\frac{e^{2} q Q}{\hbar}\right]^{2} \sum_{m=-4}^{4} \Lambda_{m}^{2-3}(\beta, \gamma) e^{i m \omega_{r} t}
$$

and

$$
\Omega_{Q, S}^{1-4}(t)=\frac{1}{\omega_{0}}\left[\frac{e^{2} q Q}{\hbar}\right]^{2} \sum_{m=-4}^{4} \Lambda_{m}^{1-4}(\beta, \gamma) e^{i m \omega_{r} t}
$$

where

$$
\begin{gathered}
\Lambda_{0}^{2-3}=-\frac{1}{32}\left(3 \cos ^{2} \beta-1\right)^{2}+\frac{33}{192} \sin ^{4} \beta \\
\Lambda_{ \pm 1}^{2-3}=\frac{1}{32 \sqrt{2}}\left[2 \sin 2 \beta\left(3 \cos ^{2} \beta-1\right)+3 \sin ^{2} \beta \sin 2 \beta\right] e^{ \pm i \gamma}
\end{gathered}
$$




$$
\begin{gathered}
\Lambda_{ \pm 2}^{2-3}=\frac{1}{32}\left[2 \sin ^{2} \beta\left(3 \cos ^{2} \beta-1\right)-3 \sin ^{2} 2 \beta\right] e^{ \pm 2 i \gamma} \\
\Lambda_{ \pm 3}^{2-3}=-\frac{1}{32 \sqrt{2}} \sin ^{2} \beta \sin 2 \beta e^{ \pm 3 i \gamma} \\
\Lambda_{ \pm 4}^{2-3}=\frac{3}{128} \sin ^{4} \beta e^{ \pm 4 i \gamma}
\end{gathered}
$$

and

$$
\begin{gathered}
\Lambda_{0}^{1-4}=\frac{10}{192}\left(3 \cos ^{2} \beta-1\right)^{2}+\frac{16}{192} \sin ^{2} 2 \beta+\frac{23}{192} \sin ^{4} \beta \\
\Lambda_{ \pm 1}^{1-4}=\frac{1}{96 \sqrt{2}}\left[\sin ^{2} \beta \sin 2 \beta-2 \sin 2 \beta\left(3 \cos ^{2} \beta-1\right)\right] e^{ \pm i \gamma} \\
\Lambda_{ \pm 2}^{1-4}=\frac{1}{96}\left[7 \sin ^{2} 2 \beta-2 \sin ^{2} \beta\left(3 \cos ^{2} \beta-1\right)\right] e^{ \pm 2 i \gamma} \\
\Lambda_{ \pm 3}^{1-4}=\frac{7}{96 \sqrt{2}} \sin ^{2} \beta \sin 2 \beta e^{ \pm 3 i \gamma} \\
\Lambda_{ \pm 4}^{1-4}=-\frac{7}{384} \sin ^{4} \beta e^{ \pm 4 i \gamma}
\end{gathered}
$$




\section{Figure Captions}

Figure 1. Powder simulations for a quadrupolar $S=3 / 2$ site $\left(C_{\mathrm{Q}}=3 \mathrm{kHz}\right.$ and $\eta_{Q}=0$; $\left.\delta_{\text {iso }}=\delta_{C S A}=0 ; v_{0}=160.4 \mathrm{MHz}\right)$ under MAS conditions $\left(v_{r}=40 \mathrm{kHz}\right)$, upon varying the sweep range $\Delta v$ and maximum field $v_{1 S}$ of a $t_{p}=8 \mathrm{~ms}$ WURST adiabatic inversion pulse. For all simulations, an ideal $90^{\circ}$ pulse was applied after the adiabatic sweep, followed by a free evolution digitization over 8.192 ms (4096 data points; $2 \mu$ dwell time) and Fourier transform to yield the spectra. The bottom row shows the ideal non-inverted MAS spectrum, denoting the single-quantum central transition (CT) without sidebands, as well as the singlequantum satellite transitions (STs) with visible spinning sidebands. A nearly ideal inversion spectrum of CT and ST (highlighted in red) is obtained only when a narrow $\Delta v(5 \mathrm{kHz})$, which does not overlap with any ST sideband(s), is applied with a low $v_{1 S}$ value $(8 \mathrm{kHz})$.

Figure 2. Inversion and spin-locking properties exhibited by the $S_{z^{-}}$and $S_{X}$-states of a spin3/2 powdered ensemble under the action of a WURST frequency-swept pulse. Included in the description of the $S_{x}$ magnetizations are the time propagations of the central- and triplequantum transition coherences (CTC and TQC) during the sweep. Simulations are presented for different quadrupolar couplings $\left(C_{Q}=e^{2} q Q / \hbar=0,1,3\right.$, and $\left.4.5 \mathrm{MHz}\right)$ with $\eta_{Q}=0$, $v_{0}=160.4 \mathrm{MHz}$, and different maximum amplitude of WURST pulse powers $\left(v_{1 S}=3,12\right.$, 40 and $80 \mathrm{kHz}$ ); but for all cases $\Delta v=10 \mathrm{kHz}$ and $t_{p}=5 \mathrm{~ms}$. The MAS spinning rate $v_{r}$ is $40 \mathrm{kHz}$, and CSA parameters are set to zero for simplicity.

Figure 3. Central transition BRAIN-CPMAS transfer dynamics for an isolated $I(1 / 2)-S(3 / 2)$ spin pair with a dipolar coupling constant of $8 \mathrm{kHz}$. Simulations were carried out with $C_{\mathrm{Q}}=$ $8 \mathrm{MHz}\left(\eta_{Q}=0\right), v_{0 I}=600 \mathrm{MHz}$, and $v_{0 S}=160.4 \mathrm{MHz}$. Time propagations of the initial $I_{\mathrm{x}}$ 
state (blue) and of the CP-enhanced $S_{\mathrm{x}}$ (green) and spin-locked $S_{\mathrm{z}}$ (red) polarizations of the S central transition are also shown. Demonstrated are (a) the $\mathrm{DQ}_{k}$ and $\mathrm{ZQ}_{k}(k=1$ or 2$) \mathrm{HH}$ conditions found as a function of $v_{1 I}$ for a fixed $v_{1 S}(8 \mathrm{kHz})$, and the (b) $\mathrm{DQ}_{1}$, (c) $\mathrm{DQ}_{2}$, (d) $\mathrm{ZQ}_{1}$, and (e) $\mathrm{ZQ}_{2}$ signal transfer dynamics. Simulations were carried out assuming onresonance irradiation for $I$ and a symmetric sweep about the $S$-spin CT second-order isotropic quadrupolar shift.

Figure 4. Experimental ${ }^{11} \mathrm{~B}$ 's CT signal intensities obtained by BRAIN-CPMAS (a,b) and ramped HH-CPMAS (c,d) experiments on a sodium tetraborate decahydrate powder spinning at $v_{r}=31 \mathrm{kHz}$, as a function of increasing ${ }^{1} \mathrm{H}(\mathrm{a}, \mathrm{c})$ and ${ }^{11} \mathrm{~B}(\mathrm{~b}, \mathrm{~d})$ rf fields (expressed in Bruker's $\mathrm{dB}$ attenuation scale). The ${ }^{11} \mathrm{~B}$ rf field was set at $v_{1 S}=4 \mathrm{kHz}$ for panel (a) and at $14 \mathrm{kHz}$ for panel (c); the ${ }^{1} \mathrm{H}$ rf field was set at $v_{1 I}=55 \mathrm{kHz}$ for panel (b) and $75 \mathrm{kHz}$ for panel (d). Swept pulse parameters for the BRAIN-CPMAS experiments were $\Delta v=25 \mathrm{kHz}$, $t_{p}=4 \mathrm{~ms}$; the ramped CPMAS experiment used a $3 \mathrm{~ms}$ long contact time. Rf fields at the local or global maxima are indicated in plain and bold font respectively, as derived from on individual $90^{\circ}$ pulse calibrations. Notice the $\mathrm{DQ}_{2}$ and $\mathrm{ZQ}_{2}$ modes yield maximum BRAINCPMAS and HH-CPMAS signal intensities respectively; these are observed at $v_{1 S}=5 \pm 3$ $\mathrm{kHz}, v_{1 I}=55 \pm 5 \mathrm{kHz}$ and $v_{1 S}=14 \pm 4 \mathrm{kHz}, v_{1 I}=75 \pm 3 \mathrm{kHz}$, respectively. The $y$-axes of the various plots are scaled relatively to the largest maximum, which has been normalized to a common value for all plots so that their relative efficiencies can be appreciated.

Figure 5. BRAIN-CPMAS, ramped CPMAS, and direct excitation MAS ${ }^{11} \mathrm{~B}$ spectra of sodium tetraborate decahydrate at spinning rates $v_{r}=31 \mathrm{kHz}$. The CP mode of each case is the $\mathrm{DQ}_{2}$ for the BRAIN-CPMAS and $\mathrm{ZQ}_{2}$ for the ramped CP methods, measured according to the optimization conditions found in Fig. 4. Experimental parameters for the two methods 
are the same as in Fig. 4. A ${ }^{11} \mathrm{~B}$ MAS spectrum was also acquired by direct excitation, via application of a $90^{\circ}$ pulse on the ${ }^{11} \mathrm{~B}$ channel and subsequent ${ }^{1} \mathrm{H}$ decoupling (Fig. 5). The relative signal intensities are indicated by the red numbers, established by placing the signal intensity of the directly excited spectrum as 100. Every spectrum was obtained by coadding 1024 transients with a 30 s recycle delay. Simulations of ideal spectra are included in the top two rows.

Figure 6. ${ }^{1} \mathrm{H}^{23} \mathrm{Na}$ BRAIN-CPMAS (red), ramped (90-110\%) CPMAS (blue), and directly excited (black) spectra of (a) sodium tetraborate decahydrate and (b) sodium citrate dihydrate at $v_{r}=25 \mathrm{kHz}$. The experimental parameters used for BRAIN-CPMAS in each case are: (a) $t_{\mathrm{p}}=4 \mathrm{~ms}, \mathrm{DQ}_{1}$ mode $\left(v_{1 S}=14 \mathrm{kHz}, v_{1 I}=4.7 \mathrm{kHz}\right)$; (b) $t_{\mathrm{p}}=2 \mathrm{~ms}, \mathrm{ZQ}_{2}$ mode $\left(v_{1 S}=30 \mathrm{kHz}\right.$, $v_{1 I}=85 \mathrm{kHz}$ ). The number on the right side of each spectrum designates relative signal intensity with respect to a directly excited spectrum with a maximum peak intensity normalized to 100 . Each spectrum was obtained by coadding 1024 transients, acquired with recycle delays of with $30 \mathrm{~s}$ (a) and $5 \mathrm{~s}$ (b). 


\section{References:}

(1) Pines, A.; Gibby, M. G.; Waugh, J. S. J. Chem. Phys. 1973, 59, 569.

(2) McDermott, A. Annu. Rev. Biophys. 2009, 38, 385.

(3) Habenstein, B.; Loquet, A. Biophys. Chem. 2016, 210, 14.

(4) Andreas, L. B.; Reese, M.; Eddy, M. T.; Gelev, V.; Ni, Q. Z; M iller, E. A.; Emsley, L.; Pintacuda, G.; Chou, J. J.; Griffin, R. G. J. Am. Chem. Soc. 2015, 137, 14877.

(5) Tycko, R. Annu. Rev. Phys. Chem. 2011 62, 279.

(6) Backhmutov, V. I. Solid-State NM R in M aterials Science: Principles and Applications; CRC Press, 2011.

(7) Reimer, J. A. Development of NM R: Solid-State NM R and M aterials Science, Post 1995; eMagRes, 2012; Vol. 1.

(8) Hartmann, S. R.; Hahn, E. L. Phys. Rev. 1902, 128, 2042.

(9) Stejskal, E. O.; Schaefer, J.; Waugh, J. S. J. Magn. Reson. 1977, $28,105$.

(10) Schaefer, J.; Stejskal, E. O. J. Am. Chem. Soc. 1976, 98, 1031.

(11) Cook, R. L.; Langford, C. H.; Yamdagni, R.; Preston, C. M. Anal. Chem. 1996, 68, 3979.

(12) Sardashti, M.; M aciel, G. E. J. Magn. Reson. 1987, 72, 467.

(13) Wind, R.; Dec, S. F.; Lock, H.; M aciel, G. E. J. Magn. Reson. 1988, 79, 136.

(14) Meier, B. H. Chem. Phys. Lett. 1992, 188, 201.

(15) Wu, X.; Zilm, K. W. J. Magn. Reson. A 1993, 104, 154.

(16) Barrie, P. J. Chem. Phys. Lett. 1993, 208, 486.

(17) Ding, S. W.; M cdowell, C. A. J. Magn. Reson. 1995, 114, 80.

(18) Amoureux, J.-P.; Pruski, M. M ol. Phys. 2002, 100, 1595.

(19) Vega, A. J. J. Magn. Reson. 1992, 96, 50.

(20) Wu, G.; Rovnyak, D.; Griffin, R. G. J. Am. Chem. Soc. 1996, 118, 9326.

(21) Dixon, I. R.; Bird, M. D.; M iller, J. R. IEEE Trans. Appl. Supercond. 2006, 16, 981.

(22) Dixon, I. R.; Adkins, T.; Ehmler, H.; M arshall, W. S.; Bird, M. D. IEEE Trans. Appl. Supercond. 2013, 23, 4300204.

(23) Man, P. P. In Encyclopedia of Nuclear Magnetic Resonance; Grant, D. M., Harris, R. K., Eds.; J. Wiley: Chichester, 1996, p 3838.

(24) Wi, S.; Gan, Z.; Schurko, R. W.; Frydman, L. J. Chem. Phys. 2015, 142, 064201.

(25) Schurko, R. W. Acc. Chem. Res. 2013, 46, 1985.

(26) Schurko, R. W. In Encyclopedia of Magnetic Resonance; Wasylishen, R. E., Ashbrook, S. E., Wimperis, S., Eds.; John Wiley \& Sons, Ltd.: 2012.

(27) MacGregor, A. W.; O'Dell, L. A.; Schurko, R. W. J. Magn. Reson. 2011, 208, 103.

(28) Harris, K. J.; Veinberg, S. L.; M ireault, C. R.; Lupulescu, A.; Frydman, L.; Schurko, R. W. Chem. Eur. J. 2013, 19, 16469.

(29) Tal, A.; Frydman, L. Prog. Nucl. Magn. Reson. Spectrosc. 2010, 57, 241.

(30) Kupce, E.; Freeman, R. J. Magn. Reson. A 1995, 115, 273.

(31) Baum, J.; Tycko, R.; Pines, A. Phys. Rev. A 1985, 32, 3435.

(32) Garwood, M.; delaBarre, L. J. Magn. Reson. 2001, 153, 155.

(33) Chandrana, C. V.; M adhub, P. K.; Wormaldc, P.; Bräuniger, T. J. Magn. Reson. 2010, 206, 255.

(34) Thakur, R. S.; Kurur, N. D.; M adhu, P. K. Chem. Phys. Lett. 2006, 426, 459.

(35) Hung, I.; Rossini, A. J.; Schurko, R. W. J. Phys. Chem. A 2004, 108, 7112.

(36) Harris, K. J.; Lupulescu, A.; Lucier, B. E. G.; Frydman, L.; Schurko, R. W. J. Magn. Reson. 2012, 224, 38.

(37) Kupce, E.; Schmidt, P.; Rance, M.; Wagner, G. J. Magn. Reson. 1998, 135, 361.

(38) Schmidt, R.; Frydman, L. Magn. Reson. Med. 2013, 70, 382. 
(39) Bohlen, J. M.; Bodenhausen, G. J. Magn. Reson. A 1993, 102, 293.

(40) O'Dell, L. A.; Schurko, R. W. Chem. Phys. Lett. 2008, 464, 97.

(41) O'Dell, L. A.; Rossini, A. J.; Schurko, R. W. Chem. Phys. Lett. 2009, 468, 330.

(42) O'Dell, L. A.; Schurko, R. W. Chem. Phys. Lett. 2008, 464, 97.

(43) O'Dell, L. A.; Schurko, R. W. J. Am. Chem. Soc. 2009, 131, 6658.

(44) Peng, W. K.; Takeda, K. J. M agn. Reson. 2007, 188, 267.

(45) Kolbert, A. C.; Bielecki, A. J. Magn. Reson. A 1995, 116, 29.

(46) Peng, W. K.; Takeda, K.; Kitagawa, M. Chem. Phys. Lett. 2006, 417, 58.

(47) M urakami, M.; Shimizu, T.; Tansho, M.; Takegoshi, K. Solid State Nucl. M agn. Reson. 2009, 36, 172.

(48) Riedel, K.; Herbst, C.; Leppert, J.; Ohlenschläger, O.; Görlach, M.; Ramachandran, R. J. Biomol. NM R 2006, 35, 275.

(49) Kervern, G.; Pintacuda, G.; Emsey, L. Chem. Phys. Lett. 2007, 435, 157.

(50) Pell, A. J.; Kervern, G.; Emsley, L.; Deschamps, M.; M assiot, D.; Grandinetti, P. J.; Pintacuda, G. J. Chem. Phys. $2011,134,024117$.

(51) MacGregor, A. W.; O'Dell, L. A.; Schurko, R. W. J. M agn. Reson. 2011, 208, 103.

(52) Wi, S.; Schurko, R. W.; Frydman, L. J. Chem. Phys. 2016, submitted.

(53) Shen, J. J. Magn. Reson. B 1996, 112, 131.

(54) Rosenfeld, D.; Panfil, S. L.; Zur, Y. J. Magn. Reson. 1997, 129, 115.

(55) Kentgens, A. P. M. J. Magn. Reson. A 1991, 95, 619.

(56) VanVeenendaal, E.; M eier, B. H.; Kentgens, A. P. M. Mol. Phys. 1998, 93, 195.

(57) Bhattacharyya, R.; Frydman, L. J. Chem. Phys. 2007, 127, 194503.

(58) Gregorovič, A.; Apih, T. J. Magn. Reson. 2013, 233, 96.

(59) Haeberlen, U. Advances in Magnetic Resonance; Academic Press: New York, 1976.

(60) Medek, A.; Harwood, J. S.; Frydman, L. J. Am. Chem. Soc. 1995, 117, 12779.

(61) Vega, S. J. Chem. Phys. 1978, 68, 5518.

(62) Vega, S.; Pines, A. J. Chem. Phys. 1977, 66, 5624.

(63) Vega, A. J. Solid State Nucl. Magn. Reson. 1992, 1, 17.

(64) Redfield, A. G. Phys. Rev. 1955, 98, 1787.

(65) Gan, Z;; Grant, D. M.; Ernst, R. R. Chem. Phys. Lett. 1996, 254, 349.

(66) Cheng, V. B.; Henry, H.; Suzukawa, J.; Wolfsberg, M. J. Chem. Phys. 1973, 59, 3992.

(67) M etz, G.; Wu, X. L.; Smith, S. O. J. Magn. Reson. A 1994, 110, 219.

(68) Peersen, O. B.; Wu, X. L.; Kustanovich, I.; Smith, S. O. J. Magn. Reson. A 1993, 104, 334.

(69) Fung, B. M.; Khitrin, A. K.; Ermolaev, K. J. Magn. Reson. 2000, 142, 97.

(70) Harris, R. K.; Nesbitt, G. J. J. Magn. Reson. 1988, 78, 245.

(71) Becerra, L. R.; Gerfen, G. J.; Temkin, R. J.; Singel, D. J.; Griffin, R. G. Phys. Rev. Lett. 1993, 71, 3561.

(72) Gerfen, G. J.; Becerra, L. R.; Hall, D. A.; Griffin, R. G.; Temkin, R. J.; Singel, D. J. J. Chem. Phys. 1995, 102, 9494.

(73) Rosay, M.; Lansing, J. C.; Haddad, K. C.; Bachovchin, W. W.; Herzfeld, J.; Temkin, R. J.; Griffin, R. G. J. Am. Chem. Soc. 2003, 125, 13626.

(74) Lesage, A.; Lelli, M.; Gajan, D.; Caporini, M. A.; Vitzthum, V.; M iéville, P.; Alauzun, J.; Roussey, A.; Thieuleux, C.; Mehdi, A.; Bodenhausen, G.; Coperet, C.; Emsley, L. J. Am. Chem. Soc. 2010, $132,15459$.

(75) Lafon, O.; Rosay, M.; Aussenac, F.; Lu, X.; Trébosc, J.; Cristini, O.; Kinowski, C.; Touati, N.; Vezin, H.; Amoureux, J.-P. Angew. Chem., Int. Ed. 2011 , 50, 8367.

(76) Lee, D.; Takahashi, H.; Thankamony, A. S. L.; Dacquin, J.-P.; Bardet, M.; Lafon, O.; Paëpe, G. D. J. Am. Chem. Soc. 2012, 134, 18491. 
(77) Blanc, F.; Sperrin, L.; Jefferson, D. A.; Pawsey, S.; Rosay, M.; Grey, C. P. J. Am. Chem. Soc. 2013, 135, 2975.

(78) Hirsh, D. A.; Rossini, A. J.; Emsley, L.; Schurko, R. W. Phys. Chem. Chem. Phys. 2016, 18, 25893. 
(a) $\Delta v=5 \mathrm{kHz}$

(b) $\Delta v=20 \mathrm{kHz}$

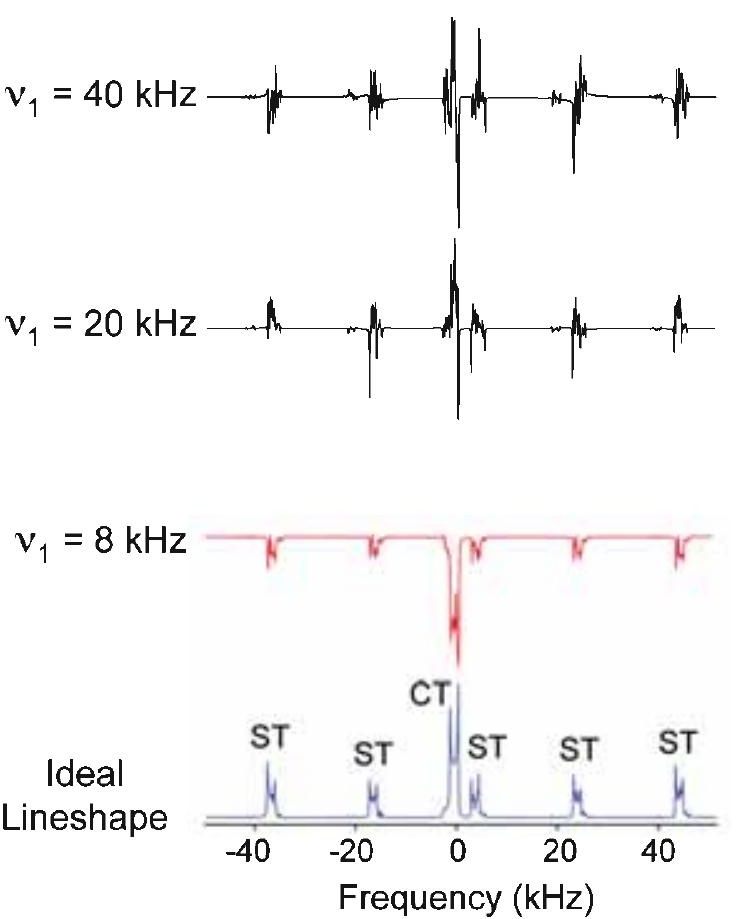

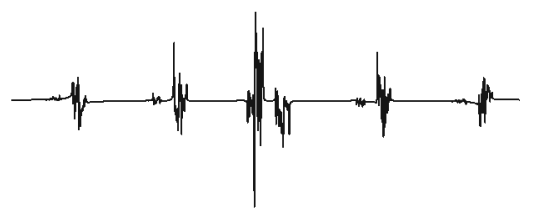
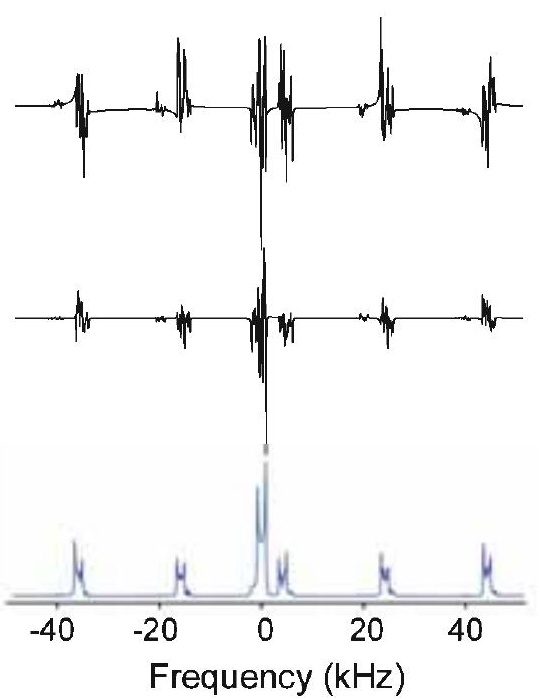

(c) $\Delta v=40 \mathrm{kHz}$
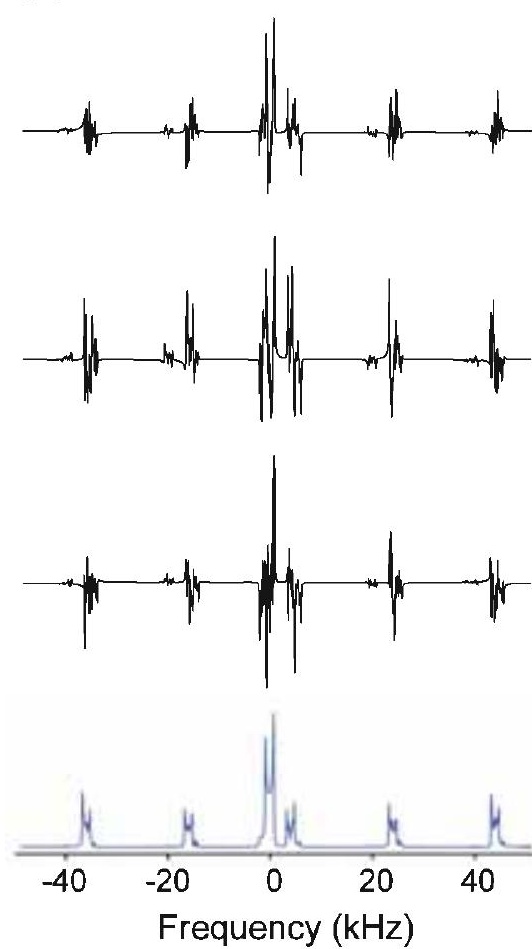


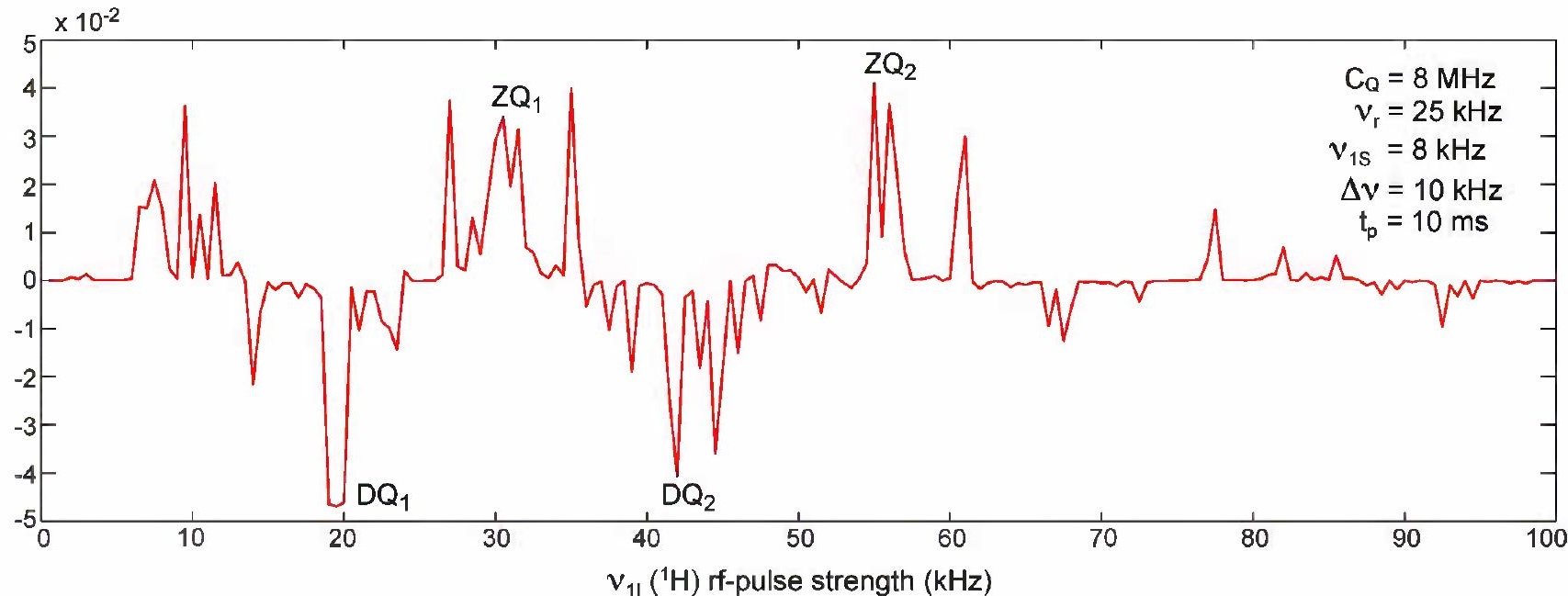

(b)

$D_{Q_{1}}$

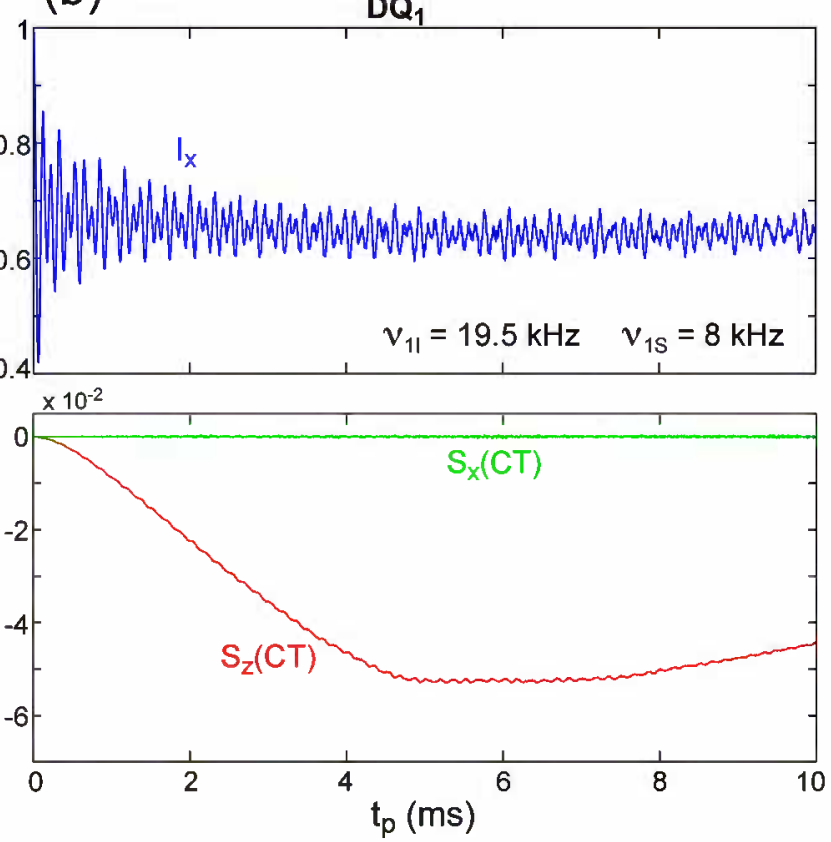

(d)

$Z Q_{1}$

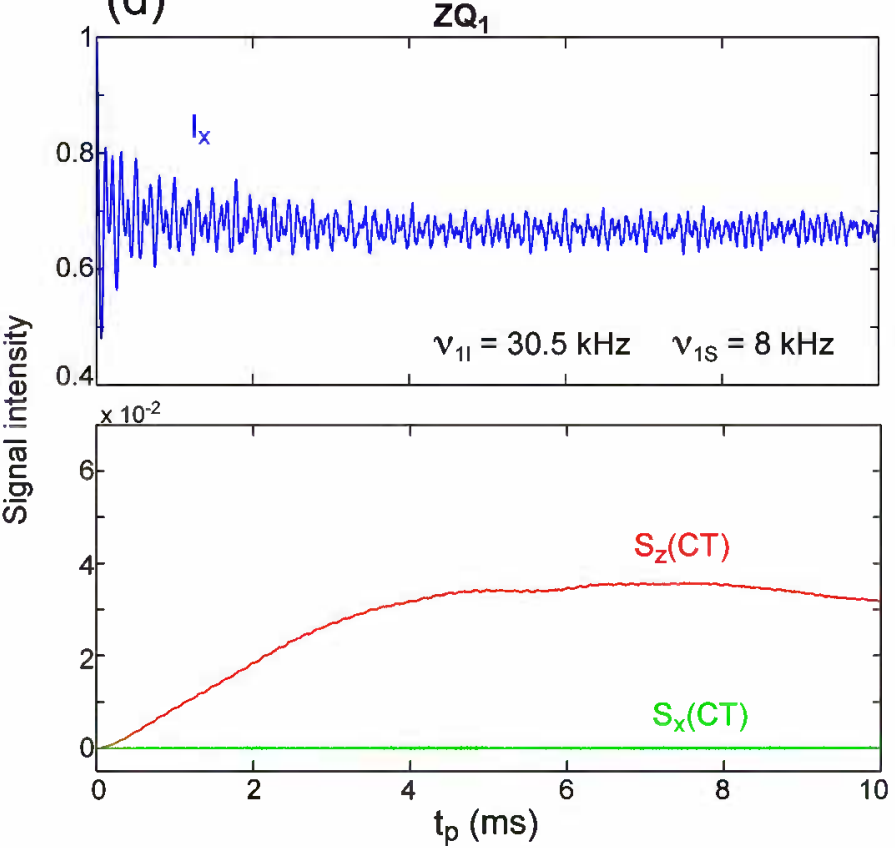

(c)

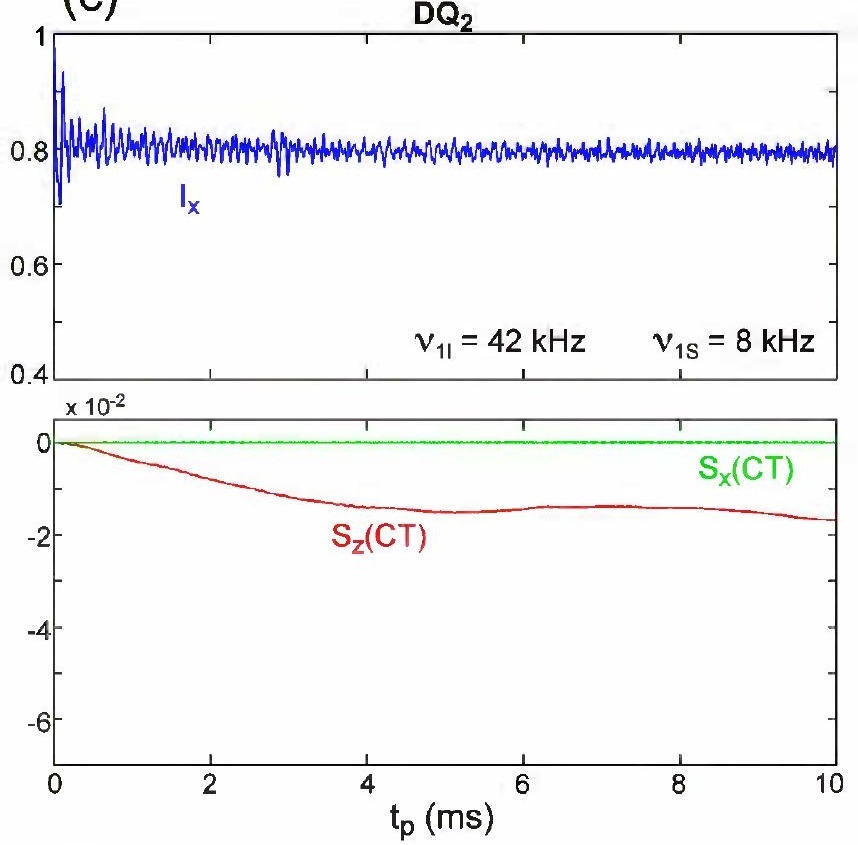

(e) $Z_{Q_{2}}$

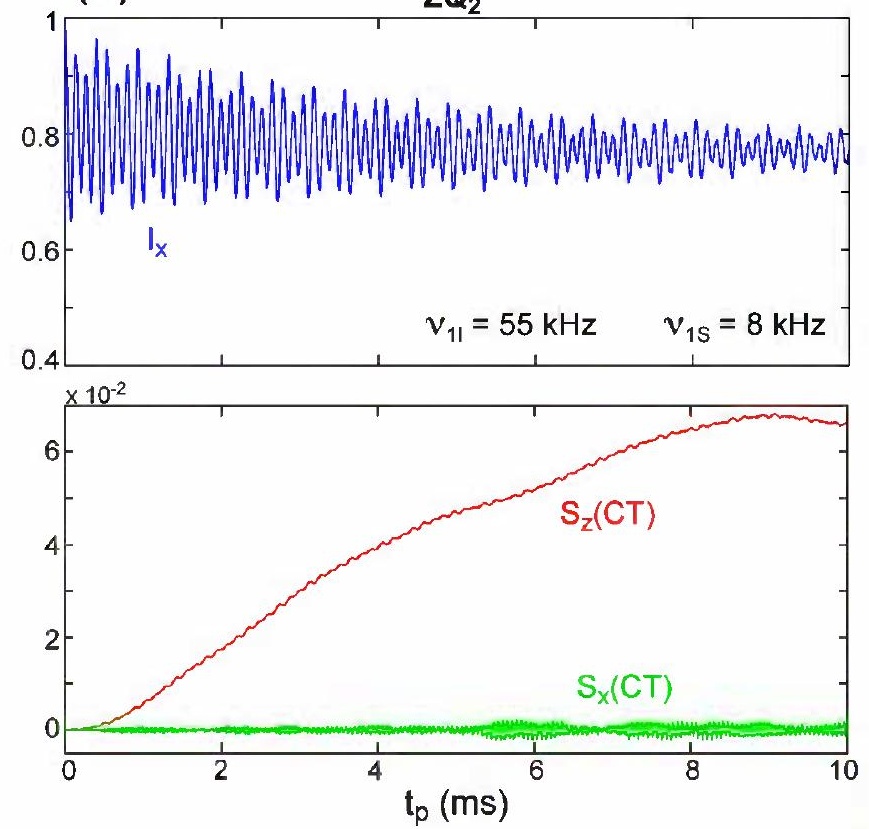


(a)

$v_{\mathrm{r}}=31 \mathrm{kHz}$

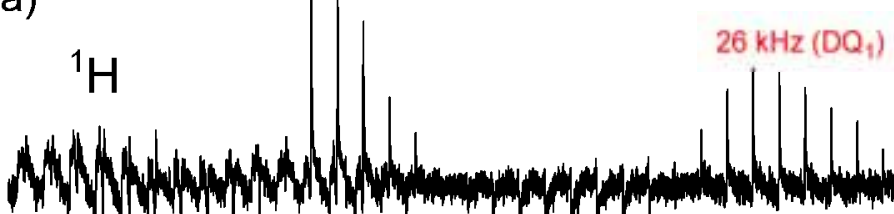
$v_{1 \mathrm{~S}}=4 \mathrm{kHz}$

(b)

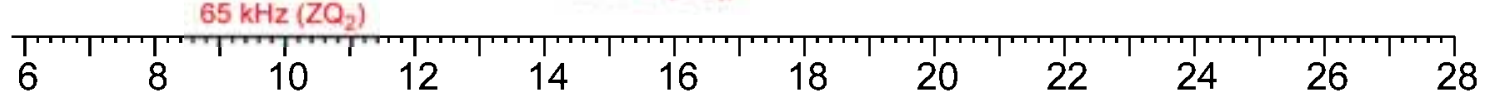

${ }^{1} \mathrm{H}$ if attenuation $(\mathrm{dB})$

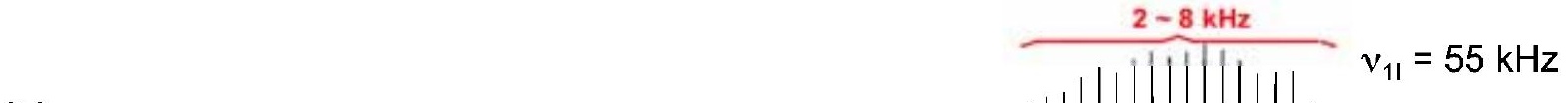

(c)

${ }^{11} \mathrm{~B}$
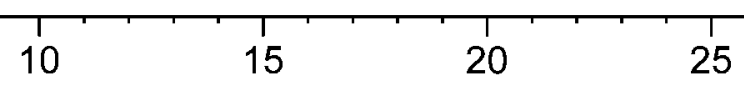

25

${ }^{11} \mathrm{~B}$ if attenuation $(\mathrm{dB})$

(c) $\quad 75 \mathrm{kHz}\left(\mathrm{ZQ}_{2}\right)$

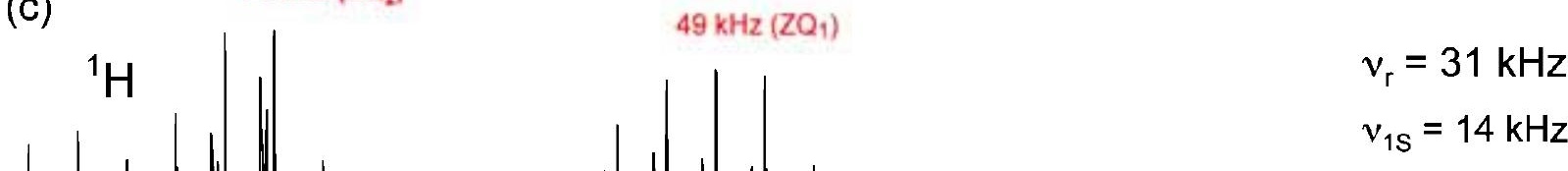

$25 \mathrm{kHz}$

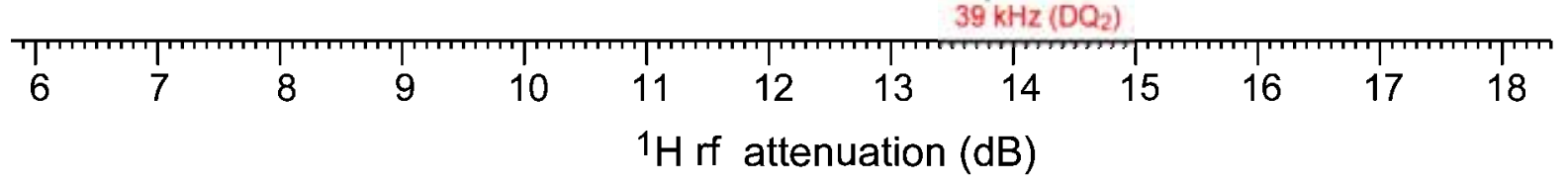

(d)

${ }^{11} \mathrm{~B}$

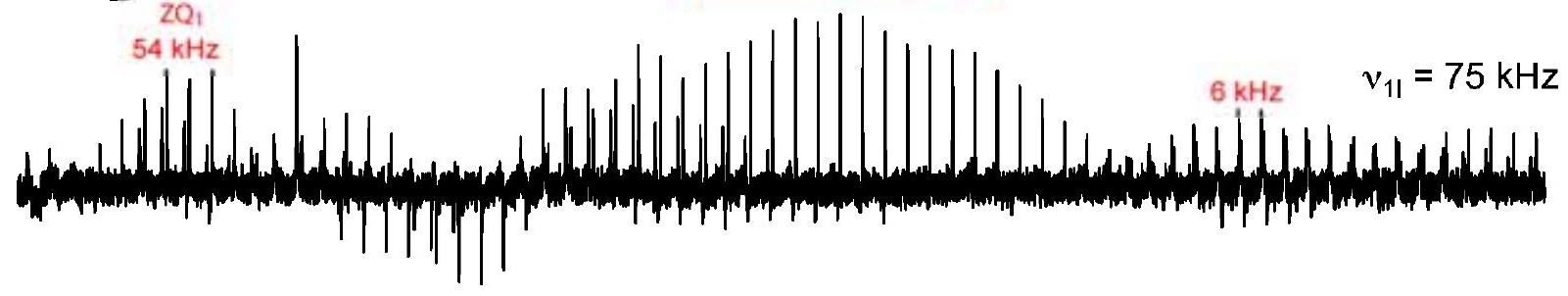

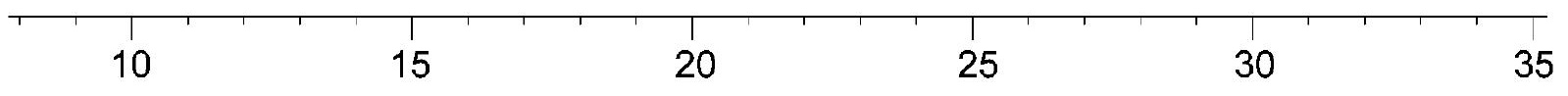

${ }^{11} \mathrm{~B}$ if attenuation $(\mathrm{dB})$ 
(a)

227

207
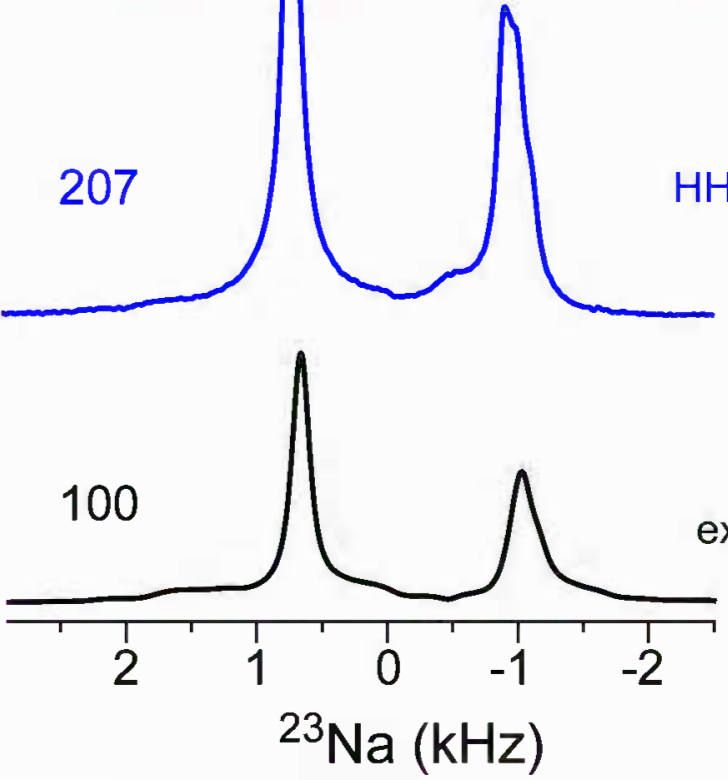

(b)

\section{BRAIN-CPMAS}

Direct

excitation 


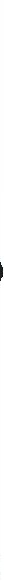

